\title{
All-electrically tunable networks of Majorana bound states
}

\author{
Song-Bo Zhang, ${ }^{1}$ Alessio Calzona, ${ }^{1}$ and Björn Trauzettel ${ }^{1,2}$ \\ ${ }^{1}$ Institute for Theoretical Physics and Astrophysics, University of Würzburg, D-97074 Würzburg, Germany \\ ${ }^{2}$ Würzburg-Dresden Cluster of Excellence ct.qmat, Germany
}

(Dated: September 8, 2020)

\begin{abstract}
Second-order topological superconductors (SOTSs) host localized Majorana fermions and provide a new platform for topological quantum computation. We propose a remarkable and feasible way to realize networks based on SOTSs which allow to nucleate and braid Majorana bound states (MBSs) in an all-electrical manner without fine-tuning. The proposed setups are scalable in a straightforward way and can accommodate any even number of MBSs. Moreover, the MBSs in the networks allow defining qubits whose states can be initialized and read out by measuring Josephson currents flowing between SOTS islands. Our proposal can be implemented in monolayers of $\mathrm{FeTe}_{1-x} \mathrm{Se}_{x}$, monolayers of $1 \mathrm{~T}^{\prime}-\mathrm{WTe}_{2}$, and inverted $\mathrm{Hg}(\mathrm{Cd}) \mathrm{Te}$ quantum wells in proximity to conventional superconductors.
\end{abstract}

Introduction. - Second-order topological superconductors (SOTSs) are characterized by topologically protected midgap bound states with zero excitation energy and codimension two [1-12]. These midgap states behave like Majorana fermions which constitute their own antiparticles [13]. They obey non-Abelian exchange statistics and could find promising applications in topological quantum computation [14-20]. Recently, SOTSs have been predicted in certain candidate systems [6-10, 2137]. Hence, they provide a feasible platform for implementing topological quantum gates [38-40]. A few theoretical proposals have been made to explore the exchange of Majorana bound states (MBSs) in SOTSs [4043]. However, they are restricted to only a single pair of MBSs or require to locally tune magnetic fields. To define a multidimensional computational ground-state manifold suitable for implementing non-Abelian quantum gates, four or more MBSs are required [16, 44]. Moreover, simpler manipulation schemes based on electrical controls are advantageous in experimental implementation and runtime for quantum gates.

In this Letter, we propose a novel way to realize electrically tunable networks of MBSs based on SOTSs. We take full advantage of the special role played by the sample geometry in SOTSs and conceive setups whose building blocks consist of isosceles right triangle islands (IRTIs) of SOTSs. By modulating local gate voltages on the islands, it is possible to nucleate an arbitrary even number of MBSs and control their positions on the networks, allowing for non-Abelian braiding. The magnetic order in our proposal can be uniform. It can, for instance, be realized by in-plane ferromagnetism (FM), antiferromagnetism (AFM), Zeeman fields, or a mixture of them. Moreover, the qubit states defined by the MBSs in the network can be initialized and readout, for instance, by measuring Josephson currents flowing between the SOTS islands. Importantly, our proposal can be implemented in a variety of candidate systems, including $1 \mathrm{~T}^{\prime}-\mathrm{WTe}_{2}$ monolayers, inverted $\mathrm{Hg}(\mathrm{Cd}) \mathrm{Te}$ quantum wells with proximity-induced superconductivity and $\mathrm{FeTe}_{1-x} \mathrm{Se}_{x}$ monolayers with intrinsic superconductivity.
MBSs on open boundaries of SOTSs.-We consider two-dimensional SOTSs which are realized by introducing $s$-wave pairing potential in combination with in-plane FM or AFM to quantum spin Hall insulators. The SOTSs can be described by

$$
\begin{aligned}
\mathcal{H}(\mathbf{k})= & m(\mathbf{k}) \tau_{z} \sigma_{z}+A \sin k_{x} s_{z} \sigma_{x}+A \sin k_{y} \tau_{z} \sigma_{y} \\
& -\mu \tau_{z}+\Delta_{0} \tau_{y} s_{y}+H_{M}
\end{aligned}
$$

in the basis $\left(c_{a \uparrow}, c_{b \uparrow}, c_{a \downarrow}, c_{b \downarrow}, c_{a \uparrow}^{\dagger}, c_{b \uparrow}^{\dagger}, c_{a \downarrow}^{\dagger}, c_{b \downarrow}^{\dagger}\right)$, where $c_{\sigma s}$ is the fermion operator with orbital (or sublattice) index $\sigma \in\{a, b\}$ and spin index $s \in\{\uparrow, \downarrow\} ; m(\mathbf{k})=2 m \cos k_{x}+$ $2 m \cos k_{y}+m_{0}-4 m$ with $m_{0} m>0 ; \mu$ is the chemical potential controllable by external gates. The Pauli matrices $\mathbf{s}, \boldsymbol{\sigma}$ and $\boldsymbol{\tau}$ act on spin, orbital and Nambu spaces, respectively. $H_{M}$ describes the magnetic order. It can be induced by close proximity to ferromagnets or antiferromagnets or by applying in-plane magnetic fields. For concreteness, we focus on the case of FM with strength $M_{0}$ in $x$ direction, $H_{M}=M_{0} \tau_{z} s_{x}$ [45].

The SOTSs feature zero-energy MBSs when open boundary conditions are enforced. To better understand this, it is instructive to derive a low-energy effective Hamiltonian on boundaries. We start with the low-energy limit of $\mathcal{H}(\mathbf{k})$ and consider the SOTSs in a disk geometry of radius $R$. In the absence of $M_{0}$ and $\Delta_{0}$, we can find helical states $\left(\Psi_{e, \uparrow}, \Psi_{e, \downarrow}, \Psi_{h, \uparrow}, \Psi_{h, \downarrow}\right)$ on the disk boundary. Using these helical states as a basis and projecting the full Hamiltonian $\mathcal{H}(\mathbf{k})$ on these states, the boundary Hamiltonian is constructed as

$$
\widetilde{\mathcal{H}}(\varphi)=-A p_{\varphi} s_{z}+\Delta_{0} \tau_{y} s_{y}-\widetilde{M} e^{-i \tau_{z} s_{z} \varphi} s_{y}-\mu \tau_{z},
$$

where $\varphi$ is the azimuthal coordinate and $p_{\varphi} \equiv-i \partial_{\varphi} / R$ the corresponding momentum defined along the boundary. The boundary states possess effective pairing potential $\Delta_{0}$ and magnetization $\widetilde{M}=M_{0} \sin \varphi$, as induced from the bulk. When $M_{0}>\bar{\Delta} \equiv\left(\Delta_{0}^{2}+\mu^{2}\right)^{1 / 2}$, we find that the energy bands of Eq. (2) change their order at the angles

$$
\varphi_{1 / 4}= \pm \arcsin \left(\bar{\Delta} / M_{0}\right), \text { and } \varphi_{2 / 3}=\varphi_{4 / 1}+\pi
$$


along the boundary. The changes of band order indicate the appearance of four MBSs $\gamma_{i}$ with $i \in\{1,2,3,4\}$, exponentially localized at $\varphi_{i}$. When they are well separated from each other, the four MBSs are at zero energy and it is possible to analytically derive their wavefunctions $\Psi_{i}$ [46]. Importantly, the chemical potential $\mu$ controls the angles $\varphi_{i}$, according to Eq. (3). This enables us to manipulate the positions of the MBSs, and eventually their fusion and braiding in an all-electrical manner, as discussed below.

Fusion properties of MBSs. - When two MBSs are brought close together, their wavefunctions start to overlap and their energies become finite. This process, known as fusion, is mediated by the electron hopping in the SOTSs. According to Eq. (1), the hopping corresponds to the operator $\hat{T}=i A\left(s_{z} \sigma_{x}+\tau_{z} \sigma_{y}\right) / 2+2 m \tau_{z} \sigma_{z}$. Thus, the fusion strength between two MBSs, say $\gamma_{i}$ and $\gamma_{j}$, can be estimated as $F_{\gamma_{i}: \gamma_{j}}=\left|\left\langle\Psi_{i}|\hat{T}| \Psi_{j}\right\rangle\right|$. On a single island, we find that the fusion strengths $F_{\gamma_{1}: \gamma_{2}}$ and $F_{\gamma_{3}: \gamma_{4}}$ are proportional to $\cos \vartheta$, while $F_{\gamma_{1}: \gamma_{4}}$ and $F_{\gamma_{2}: \gamma_{3}}$ to $\sin \vartheta$, where $\vartheta=\arctan \left(\mu / \Delta_{0}\right)$. By contrast, the fusion between $\gamma_{1}$ and $\gamma_{3}$ (or $\gamma_{2}$ and $\gamma_{4}$ ) is strictly forbidden, due to inversion symmetry of the SOTSs [46].

The fusion properties become richer when we consider two sets of MBSs $\left\{\gamma_{i}\right\}$ and $\left\{\gamma_{i}^{\prime}\right\}$ (with $i \in\{1,2,3,4\}$ ) belonging to two different islands, featuring a finite pairing phase difference. In this case, when two MBSs from different islands are brought close together, they can always fuse in general. The mutual fusion strengths $F_{\gamma_{i}: \gamma_{j}^{\prime}}$ are summarized in Table I and depend sinusoidally on the pairing phase difference $2 \delta \Phi$ and the chemical potentials $\mu$ and $\mu^{\prime}$ of the two islands.

\begin{tabular}{|c||c|c|c|c|}
\hline & $\gamma_{1}$ & $\gamma_{2}$ & $\gamma_{3}$ & $\gamma_{4}$ \\
\hline$\gamma_{1}^{\prime}$ & $\sin \vartheta_{-} \sin \delta \Phi$ & $\cos \vartheta_{+} \cos \delta \Phi$ & $\cos \vartheta_{-} \sin \delta \Phi$ & $\sin \vartheta_{+} \cos \delta \Phi$ \\
\hline$\gamma_{2}^{\prime}$ & $\cos \vartheta_{+} \cos \delta \Phi$ & $\sin \vartheta_{-} \sin \delta \Phi$ & $\sin \vartheta_{+} \cos \delta \Phi$ & $\cos \vartheta_{-} \sin \delta \Phi$ \\
\hline$\gamma_{3}^{\prime}$ & $\cos \vartheta_{-} \sin \delta \Phi$ & $\sin \vartheta_{+} \cos \delta \Phi$ & $\sin \vartheta_{-} \sin \delta \Phi$ & $\cos \vartheta_{+} \cos \delta \Phi$ \\
\hline$\gamma_{4}^{\prime}$ & $\sin \vartheta_{+} \cos \delta \Phi$ & $\cos \vartheta_{-} \sin \delta \Phi$ & $\cos \vartheta_{+} \cos \delta \Phi$ & $\sin \vartheta_{-} \sin \delta \Phi$ \\
\hline
\end{tabular}

Table I. Fusion strength $F_{\gamma_{i}: \gamma_{i^{\prime}}}$ of MBSs $\left\{\gamma_{i}\right\}$ and $\left\{\gamma_{i}^{\prime}\right\}$ belonging to two SOTS islands. The table displays the dependence of $F_{\gamma_{i}: \gamma_{i^{\prime}}}$ on $\mu$ and $\mu^{\prime}$ and on $\delta \Phi$. We define $\vartheta_{ \pm}=\left(\vartheta \pm \vartheta^{\prime}\right) / 2$, $\vartheta=\arctan \left(\mu / \Delta_{0}\right)$ and $\vartheta^{\prime}=\arctan \left(\mu^{\prime} / \Delta_{0}\right)$. Results for the fusion of MBSs belonging to the same island can be obtained by taking $\gamma_{i}^{\prime}=\gamma_{i}, \delta \Phi=0$ and $\mu^{\prime}=\mu$.

Manipulation of MBSs in IRTIs.-In order to obtain a scalable platform hosting any even number of MBSs which are manipulable by purely electrical means, it is essential to go beyond the simple disk geometry presented so far. Particularly, we focus on IRTIs, the short sides of which are orientated in $x$ and $y$ directions, as depicted in Fig. 1. To develop some intuition about the appearance of MBSs in the IRTIs, one can relate the latter to the disk geometry in the following way: the dotted lines normal to the triangle sides define three arcs of the disk

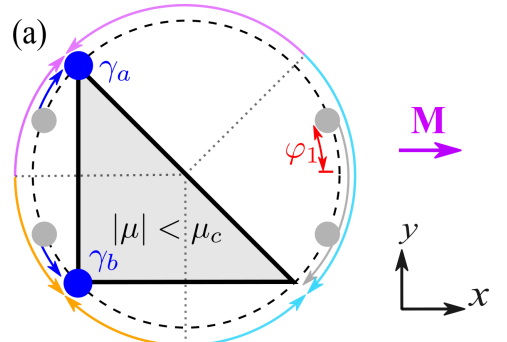

(c)
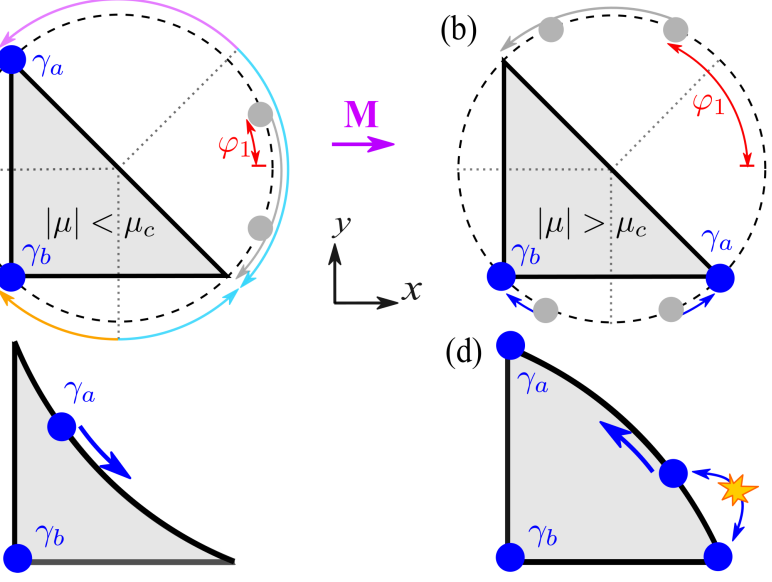

(d)

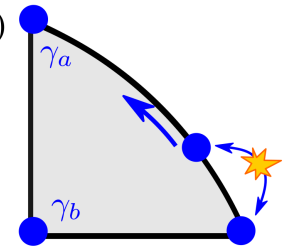

Fig. 1. Positions of two MBSs (blue dots) in an IRTI for (a) $|\mu|<\mu_{c}$ and (b) $|\mu|>\mu_{c}$, respectively. The gray dots denote the four MBSs on a disk boundary (dashed curve). The magnetic order $(\mathbf{M})$ is fixed in $x$ direction. By increasing $\mu$ from $0<\mu<\mu_{c}$ to $\mu>\mu_{c}$, the angle $\varphi_{1}$ (in red) increases and one MBS is moved from one sharp-angle vertex to the other one. Schematics of IRTIs with small concavity (c) or convexity (d) on the diagonals.

boundary (dashed curves); all the points belonging to the same arc reduce to the corresponding vertex of the triangle (colored arrows); conversely, each side of the triangle reduces to a single point on the disk. Out of the four MBSs (gray dots) hosted by the disk, two of them must locate on the same arc meaning that, in the triangle, they fuse on the same vertex. By contrast, the two remaining MBSs locate on different arcs and thus stay robustly as zero-energy corner states (blue dots) in the IRTI. Which vertices host the MBSs crucially depends on the angles $\varphi_{i}\left(\varphi_{1}\right.$ is depicted in red $)$ and, therefore, on the value of the chemical potential $\mu$.

For $|\mu|<\mu_{c} \equiv\left(M_{0}^{2} / 2-\Delta_{0}^{2}\right)^{1 / 2}$, the four MBSs on the disk are sketched in Fig. 1(a). For $|\mu|>\mu_{c}$, the MBSs are located as shown in Fig. 1(b). By slowly tuning $\mu$ across $\mu_{c}$, say from $\mu_{\mathrm{d}}\left(<\mu_{c}\right)$ to $\mu_{\mathrm{u}}\left(>\mu_{c}\right)$, we can thus adiabatically move one MBS between two sharp-angle vortices while the other one stays fixed at the right-angle vertex. We observe that a finite $\mu_{c}$ requires $M_{0}>\sqrt{2} \Delta_{0}$. When $\mu$ is close to $\mu_{c}$, the localization length of the movable MBS along the diagonal is approximately proportional to $A \Delta_{0} /\left(\mu_{c}\left|\mu-\mu_{c}\right|\right)$. Therefore, larger islands pose weaker constraints on the difference $\mu_{\mathrm{u}}-\mu_{\mathrm{d}}$. The possibility to move MBSs between two vertices is confirmed numerically [46]. These results apply to any IRTIs with the short sides in $x$ and $y$ directions.

To get more insights into the fundamental role played by the SOTS geometry and to make further use of it, we consider a small bending on the diagonal. Interestingly, we find that small concavity on the diagonal allows us to smoothly move the MBS along the diagonal [Fig. 1(c)]. It also helps to enhance the excitation gap that protects 
(a)
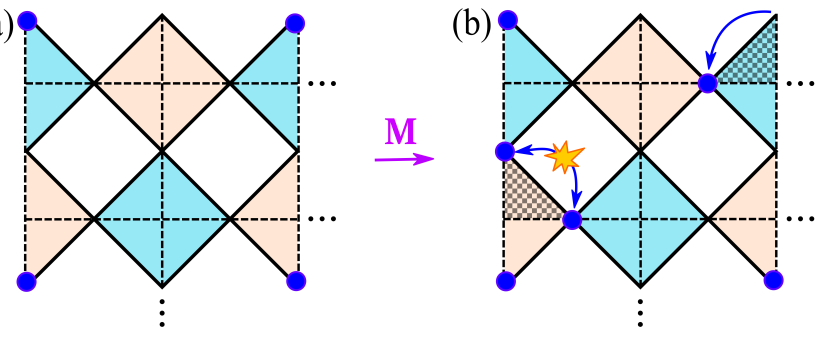

Fig. 2. Networks of connected IRTIs. The cyan and yellow colors distinguish between two pairing phases on the islands. The white regions are vacuum or trivial insulator. The dashed lines mark the boundaries between the IRTIs. In (a), all $\mu_{j}=$ $\mu_{\mathrm{d}}$ and the network hosts four MBSs indicated by the blue dots. In (b), the chemical potentials of triangles marked by the shadow pattern have been tuned to $\mu_{\mathrm{u}}$, resulting in the movement of the top-right MBS and in the nucleation of two additional MBSs.

the MBSs since the diagonal becomes fully gapped everywhere except for one point in space even at $\mu_{c}$ [46]. By contrast, small convexity tends to nucleate an extra Majorana pair and thus momentarily increases the ground-state degeneracy [Fig. 1(d)].

Building networks of MBSs.-By properly connecting several IRTIs, networks of diagonals can be defined, for instance, as sketched in Fig. 2 (more examples are given in the Supplemental Material [46]). When two or more vertices get in contact, there is a finite overlap between the wavefunctions of different MBSs, which fuse according to the inter- and intra-island fusion strengths summarized in Table I. The latter clearly depends on the chemical potential and the superconducting phases $\Phi_{j}$ of adjacent IRTIs. For concreteness, in the following, we focus on the configuration illustrated in Fig. 2, where we apply $\Phi_{j}=0$ for the cyan triangles and $\Phi_{j}=\Phi_{0} \neq p \pi$ (with $p \in \mathbb{Z}$ ) for the yellow ones. As a result, we observe that every time an even number of MBSs approach the same point, they completely fuse. Conversely, when an odd number of MBSs approach the same point, a single MBS is left at zero energy.

By tuning the chemical potentials of individual IRTIs across $\mu_{c}$, it is therefore possible to either nucleate, fuse, or move MBSs on the network. Two clarifying examples are illustrated in Fig. 2. In Fig. 2(a), all chemical potentials are set to $\mu_{\mathrm{d}}<\mu_{c}$, resulting in the presence of four MBSs. In Fig. 2(b), the chemical potentials of two IRTIs (highlighted by shadow pattern) have been tuned to $\mu_{\mathrm{u}}>\mu_{c}$. Consequently, the top-right MBS is moved while a new pair of MBSs has been nucleated in the left-bottom of the network.

It is important to stress that the Majorana manipulation does not rely on fine-tuning of parameters. The proposed setup can therefore be easily scaled up, just by adding more IRTIs, in order to accommodate an arbitrary number of MBSs. Since each MBS is exponentially

localized on a specific node of the network, the lifting of the ground-state degeneracy is exponentially small in the size of each island.

Braiding a Majorana qubit.-To illustrate the capabilities of our networks, we now show how to braid a couple of MBSs, thus implementing a phase gate on a Majorana qubit. The latter consists of four MBSs, which can be hosted by the six-island structure depicted in Fig. 3. We label the IRTIs by $T_{j}$ (with $j \in\{1, \cdots, 6\}$ ) and the corresponding chemical potentials and superconducting phases by $\mu_{j}$ and $\Phi_{j}$, respectively. For the numerical simulation illustrated in Fig. 3 , we considered $\Phi_{5}=\Phi_{6}=\pi / 2$ and $\Phi_{j}=0$ otherwise.

The initial configuration, Fig. 3(a), features $\mu_{j}=\mu_{\mathrm{u}}$ for $j \in\{1,5,6\}$ and $\mu_{j}=\mu_{\mathrm{d}}$ otherwise. We can observe four MBSs which are indicated by the black localized densities and labeled by $\gamma_{a}, \gamma_{b}, \gamma_{c}$ and $\gamma_{d}$. In order to braid $\gamma_{a}$ and $\gamma_{b}$, the chemical potentials $\mu_{4}, \mu_{5}$ and $\mu_{6}$ must be adiabatically tuned in time, according to Fig. $3(\mathrm{i})$. This results in the motion of $\gamma_{a}$ and $\gamma_{b}$ along the

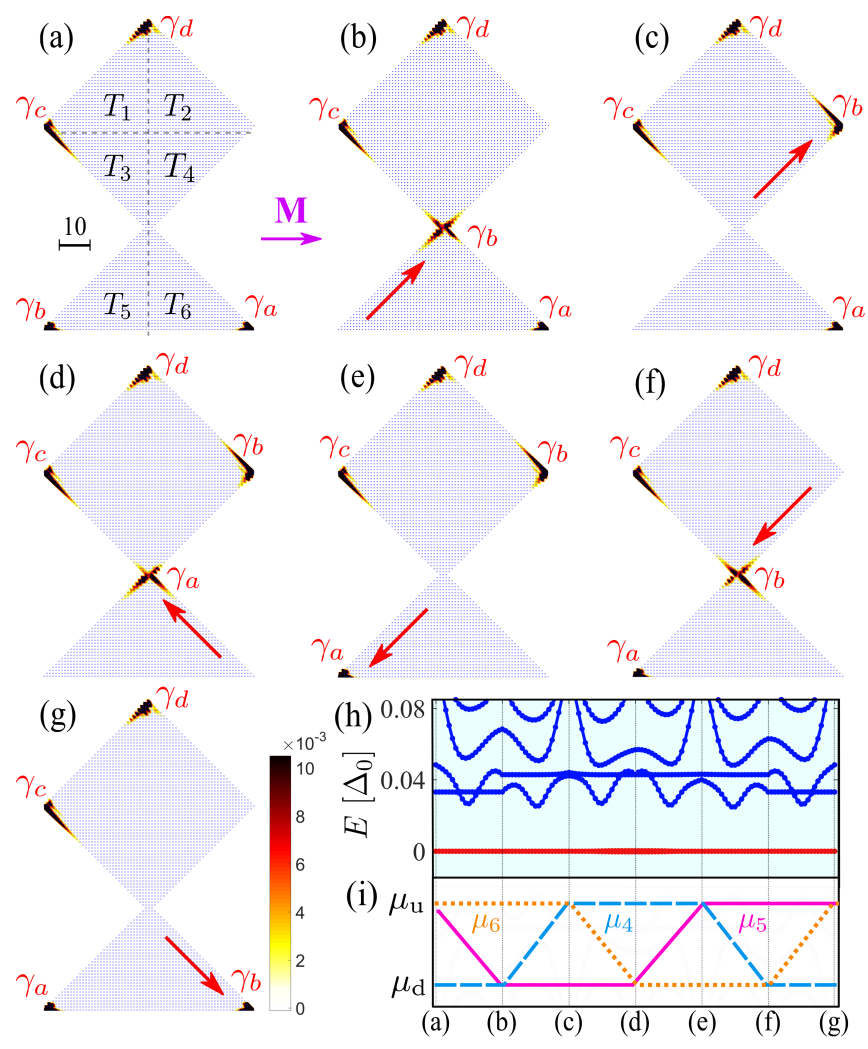

Fig. 3. Numerical simulation of braiding $\gamma_{a}$ and $\gamma_{b}$. (a)-(g) Seven subsequent snapshots show the positions of the four MBSs (black localized densities). During the protocol, $\mu_{4}$, $\mu_{5}$ and $\mu_{6}$ are varied in time, according to (i), while $\mu_{1}=\mu_{\mathrm{u}}$ and $\mu_{2}=\mu_{3}=\mu_{\mathrm{d}}$ are fixed. (h) The energy spectrum of the system during the process. It is symmetric with respect to zero energy. The parameters are $\mu_{\mathrm{u}}=0.15 m_{0}, \mu_{\mathrm{d}}=0.05 m_{0}$, $M_{0}=0.4 m_{0}, \Delta_{0}=0.25 m_{0}$, and $A=m=0.5 m_{0}$, the shortside length of the IRTIs is $L=35 a$. 
diagonals of $T_{4}, T_{5}$ and $T_{6}$, as shown in Figs. 3 (a)-(g). At the end of the protocol, while the system has the same parameters as in the initial state, the positions of $\gamma_{a}$ and $\gamma_{b}$ are exchanged. Importantly, during the whole process, the four MBSs stay robustly at zero energy [red bands in Fig. 3(h)]. They are always separated from excited states (blue bands) by an energy gap. Similar procedures apply to exchange other MBS pairs [46].
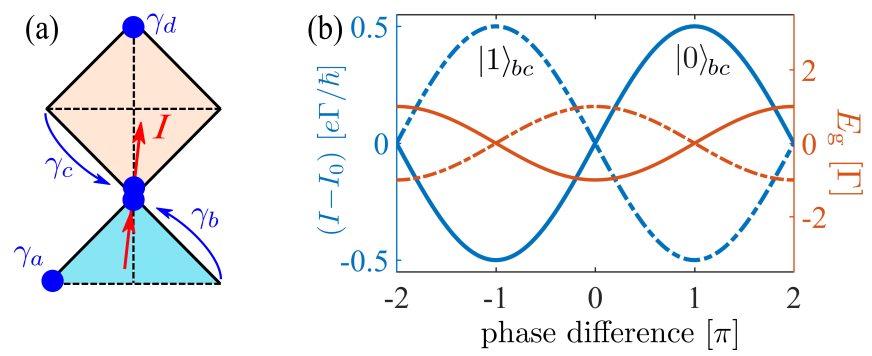

Fig. 4. (a) Detection of the parity $P_{b c}$ of $\gamma_{b}$ and $\gamma_{c}$ by measuring the Josephson current. (b) Ground-state energy $E_{g}$ (orange curves) of the two coupled MBSs, $\gamma_{b}$ and $\gamma_{c}$, and the Josephson current (blue curves) across the junction as functions of the pairing phase difference. Solid and broken curves correspond to the two parity states, $P_{b c}|0\rangle_{b c}=|0\rangle_{b c}$ and $P_{b c}\left|1_{b c}\right\rangle=-\left|1_{b c}\right\rangle$.

Because of the non-Abelian nature of MBSs, the braiding of $\gamma_{a}$ and $\gamma_{b}$ results in a nontrivial unitary operation $U_{a b}=\exp \left(\pi \gamma_{a} \gamma_{b} / 4\right)$ on the Majorana qubit [14]. It corresponds to a quantum gate that implements a $\pi / 2$ rotation on the Bloch sphere. This can be experimentally confirmed by measuring the parity of two different couples of MBSs, $P_{b c}=i \gamma_{b} \gamma_{c}$ and $P_{a c}=i \gamma_{a} \gamma_{c}$. The former one can be used to initialize the qubit, say in the eigenstate of $P_{b c}|0\rangle=|0\rangle$. Then, the braiding rotates the initial state to $U_{a b}|0\rangle$ which is an eigenstate of $P_{a c} U_{a b}|0\rangle=U_{a b}|0\rangle$. The validity of this result can be straightforwardly verified by measuring $P_{a c}$.

Remarkably, our all-in-one setup allows for initialization, braiding, and readout. Indeed, because of the possibility to move and fuse arbitrary couples of MBSs on the network, we can measure a generic parity operator $P_{\alpha \beta}$. For concreteness, we describe the measurement of $P_{b c}$ in the six-island architecture. In this case, one must fuse $\gamma_{b}$ and $\gamma_{c}$ by moving them in the region which defines a Josephson junction between islands with different pairing phases [Fig. 4(a)]. The effective Hamiltonian which describes the coupling between the two MBSs reads $\mathcal{H}_{b c}=\Gamma \cos (\delta \Phi) P_{b c}$, where $2 \delta \Phi$ is the pairing phase difference and $\Gamma$ is the coupling strength that depends on the chemical potentials and wavefunction overlap. The two eigenenergies are therefore $E_{g}= \pm \Gamma \cos (\delta \Phi)$ [orange curves in Fig. 4(b)]. At zero temperature, the Josephson current across the junction is $I=I_{0} \mp e \Gamma \sin (\delta \Phi) / 2 \hbar=$ $I_{0} \mp I_{\mathrm{mbs}}$, where $I_{\mathrm{mbs}}$ and $I_{0}$ are the contributions from the MBSs and ordinary fermions, respectively [47]. As long as $2 \delta \Phi \neq 0$, by probing $I$ flowing between the islands one can therefore measure $P_{b c}$ [Fig. 4(b)]. In principle, other measurement schemes based on quantum dots are also possible [46, 49].

Experimental feasibility and summary.-Remarkably, $\mathrm{FeTe}_{1-x} \mathrm{Se}_{x}$ monolayers have been shown to possess a band inversion at the $\Gamma$ point [50-52] and intrinsic hightemperature superconductivity [53, 54]. The magnetic order may be induced by putting (anti)ferromagnets, e.g., FeSe or FeTe layers [55-57], on top of $\mathrm{FeTe}_{1-x} \mathrm{Se}_{x}$ monolayers or by applying in-plane magnetic fields. We note that the sustenance of superconductivity under strong inplane magnetic fields in this material has been reported experimentally [58]. Interestingly, FeSe monolayers coupling to substrates may have all the desired ingredients for realizing SOTSs (namely, band inversions at the $M$ points, superconductivity [59-61] and AFM order [62]) intrinsically within one material. Quantum spin Hall insulators, such as monolayers of $1 \mathrm{~T}^{\prime}-\mathrm{WTe}_{2}$ [63-69], inverted $\mathrm{Hg}(\mathrm{Cd}) \mathrm{Te}$ and InAs/GaSb quantum wells [70-76], in proximity to conventional superconductors could offer another candidate system. Notably, electric gating on superconducting $1 \mathrm{~T}^{\prime}-\mathrm{WTe}_{2}$ monolayers has already been demonstrated [67, 68].

In general, the control of local chemical potentials on the islands might be a challenging task. However, it is by no means necessary to fine tune the chemical potentials to specific values of $\mu_{\mathrm{u}}$ and $\mu_{\mathrm{d}}$. The only requirements are (i) the possibility to tune $\mu$ across its critical value, i.e., $\mu_{\mathrm{d}}<\mu_{c}<\mu_{\mathrm{u}}$ and (ii) that, at $\mu_{\mathrm{u}}$ and $\mu_{\mathrm{d}}$, the MBSs are well localized at the vertices of IRTIs. Importantly, we numerically prove that inhomogeneities of chemical potential within each IRTI are not detrimental to our proposal [46]. Finally, we remark that field effects on (superconducting) thin films have proven to be a valid alternative to conventional chemical doping in order to tune the carrier density [77-79], suggesting the feasibility of controlling local chemical potentials with external gates.

An important issue, when it comes to Majorana-based quantum computation, is represented by quasiparticle poisoning (QP) [80-82], causing detrimental flips in the total fermion parity of individual qubits. In this respect, the large superconducting gap of $\mathrm{FeTe}_{1-x} \mathrm{Se}_{x}$ monolayers (up to $16.5 \mathrm{meV}$ [53]) represents a prime advantage: (i) It is likely to decrease the QP rate. (ii) It allows for faster adiabatic qubit operations. Moreover, it might be possible to implement quasiparticle filters which have proven, at least for quantum wires, to increase the characteristic QP time up to $(1 / 200) \mathrm{s}$ [83].

In summary, we have proposed a feasible way to realize networks of SOTSs which can accommodate any even number of topologically protected MBSs. The MBSs can be generated, moved and fused by all-electrical means. Our proposal allows to define a qubit, braid the corresponding MBSs, and measure the nontrivial outcome of this operation. 
We thank Sang-Jun Choi, Ning Hao, Tobias Kiessling, and Wenbin Rui for valuable discussion. This work was supported by the DFG (SPP1666 and SFB1170 "ToCoTronics"), the Würzburg-Dresden Cluster of Excellence ct.qmat, EXC2147, project-id 390858490, and the Elitenetzwerk Bayern Graduate School on "Topological Insulators".

S.B.Z. and A.C. contributed equally to this work.

[1] J. Langbehn, Y. Peng, L. Trifunovic, F. von Oppen, and P. W. Brouwer, "Reflection-Symmetric Second-Order Topological Insulators and Superconductors," Phys. Rev. Lett. 119, 246401 (2017).

[2] W. A. Benalcazar, B. A. Bernevig, and T. L. Hughes, "Quantized electric multipole insulators," Science 357, 61 (2017).

[3] Z. Song, Z. Fang, and C. Fang, "( $d-2)$-Dimensional Edge States of Rotation Symmetry Protected Topological States," Phys. Rev. Lett. 119, 246402 (2017).

[4] W. A. Benalcazar, B. A. Bernevig, and T. L. Hughes, "Electric multipole moments, topological multipole moment pumping, and chiral hinge states in crystalline insulators," Phys. Rev. B 96, 245115 (2017).

[5] F. Schindler, A. M. Cook, M. G. Vergniory, Z. Wang, S. S. P. Parkin, B. A. Bernevig, and T. Neupert, "Higherorder topological insulators," Sci. Adv. 4, eaat0346 (2018).

[6] Q. Wang, C.-C. Liu, Y.-M. Lu, and F. Zhang, "HighTemperature Majorana Corner States," Phys. Rev. Lett. 121, 186801 (2018).

[7] Z. Yan, F. Song, and Z. Wang, "Majorana Corner Modes in a High-Temperature Platform," Phys. Rev. Lett. 121, 096803 (2018).

[8] T. Liu, J. J. He, and F. Nori, "Majorana corner states in a two-dimensional magnetic topological insulator on a high-temperature superconductor," Phys. Rev. B 98, 245413 (2018).

[9] M. Geier, L. Trifunovic, M. Hoskam, and P. W. Brouwer, "Second-order topological insulators and superconductors with an order-two crystalline symmetry," Phys. Rev. B 97, 205135 (2018).

[10] H. Shapourian, Y. Wang, and S. Ryu, "Topological crystalline superconductivity and second-order topological superconductivity in nodal-loop materials," Phys. Rev. B 97, 094508 (2018).

[11] A. Skurativska, T. Neupert, and M. H. Fischer, "Atomic limit and inversion-symmetry indicators for topological superconductors," Phys. Rev. Res. 2, 013064 (2020).

[12] A. Tiwari, M.-H. Li, B. A. Bernevig, T. Neupert, and S. A. Parameswaran, "Unhinging the Surfaces of Higher-Order Topological Insulators and Superconductors," Phys. Rev. Lett. 124, 046801 (2020).

[13] E. Majorana, "Teoria simmetrica dell'elettrone e del positrone," Nuovo Cimento 14, 171-184 (1937).

[14] D. A. Ivanov, "Non-Abelian Statistics of Half-Quantum Vortices in $p$-wave Superconductors," Phys. Rev. Lett. 86, 268-271 (2001).

[15] A. Y. Kitaev, "Fault-tolerant quantum computation by anyons," Ann. Phys. 303, 2-30 (2003).
[16] C. Nayak, S. H. Simon, A. Stern, M. Freedman, and S. Das Sarma, "Non-Abelian anyons and topological quantum computation," Rev. Mod. Phys. 80, 1083 (2008).

[17] J. Alicea, "New directions in the pursuit of Majorana fermions in solid state systems," Rep. Prog. Phys. 75, 076501 (2012).

[18] C. W. J. Beenakker, "Search for Majorana fermions in superconductors," Annu. Rev. Condens. Matter Phys. 4, 113 (2013).

[19] S. Das Sarma, M. Freedman, and C. Nayak, "Majorana zero modes and topological quantum computation," npj Quantum Inf. 1, 15001 (2015).

[20] S. R. Elliott and M. Franz, "Colloquium: Majorana fermions in nuclear, particle, and solid-state physics," Rev. Mod. Phys. 87, 137 (2015).

[21] C.-H. Hsu, P. Stano, J. Klinovaja, and D. Loss, "Majorana Kramers Pairs in Higher-Order Topological Insulators," Phys. Rev. Lett. 121, 196801 (2018).

[22] Y. Volpez, D. Loss, and J. Klinovaja, "Second-Order Topological Superconductivity in $\pi$-Junction Rashba Layers," Phys. Rev. Lett. 122, 126402 (2019).

[23] S.-B. Zhang and B. Trauzettel, "Detection of secondorder topological superconductors by Josephson junctions," Phys. Rev. Res. 2, 012018 (2020).

[24] S. A. A. Ghorashi, X. Hu, T. L. Hughes, and E. Rossi, "Second-order Dirac superconductors and magnetic field induced Majorana hinge modes," Phys. Rev. B 100, 020509 (2019).

[25] R.-X. Zhang, W. S. Cole, X. Wu, and S. Das Sarma, "Higher-Order Topology and Nodal Topological Superconductivity in Fe(Se,Te) Heterostructures," Phys. Rev. Lett. 123, 167001 (2019).

[26] K. Plekhanov, M. Thakurathi, D. Loss, and J. Klinovaja, "Floquet second-order topological superconductor driven via ferromagnetic resonance," Phys. Rev. Res. 1, 032013 (2019).

[27] J. Ahn and B.-J. Yang, "Higher-order topological superconductivity of spin-Polarized fermions," Phys. Rev. Res. 2, 012060 (2020).

[28] S. Franca, D. V. Efremov, and I. C. Fulga, "Phasetunable second-order topological superconductor," Phys. Rev. B 100, 075415 (2019).

[29] R.-X. Zhang, W. S. Cole, and S. Das Sarma, "Helical Hinge Majorana Modes in Iron-Based Superconductors," Phys. Rev. Lett. 122, 187001 (2019).

[30] N. Bultinck, B. A. Bernevig, and M. P. Zaletel, "Threedimensional superconductors with hybrid higher-order topology," Phys. Rev. B 99, 125149 (2019).

[31] Y.-T. Hsu, W. S. Cole, R.-X. Zhang, and J. D. Sau, "Inversion-Protected Higher-Order Topological Superconductivity in Monolayer WTe $\mathrm{WT}_{2}$,"Phys. Rev. Lett. 125, 097001 (2020).

[32] Z. Yan, "Higher-Order Topological Odd-Parity Superconductors," Phys. Rev. Lett. 123, 177001 (2019).

[33] X.-H. Pan, K.-J. Yang, L. Chen, G. Xu, C.-X. Liu, and X. Liu, "Lattice-Symmetry-Assisted Second-Order Topological Superconductors and Majorana Patterns," Phys. Rev. Lett. 123, 156801 (2019).

[34] Y. Peng, "Floquet higher-order topological insulators and superconductors with space-time symmetries," Phys. Rev. Research 2, 013124 (2020).

[35] Y.-J. Wu, J. Hou, Y..-M. Li, X.-W. Luo, X. Shi, and C. Zhang, "In-Plane Zeeman-Field-Induced Majorana 
Corner and Hinge Modes in an $s$-Wave Superconductor Heterostructure," Phys. Rev. Lett. 124, 227001 (2020).

[36] K. Laubscher, D. Loss, and J. Klinovaja, "Majorana and parafermion corner states from two coupled sheets of bilayer graphene," Phys. Rev. Res. 2, 013330 (2020).

[37] X. Wu, X. Liu, R. Thomale, and C.-X. Liu, "High$T_{c}$ Superconductor $\mathrm{Fe}(\mathrm{Se}, \mathrm{Te})$ Monolayer: an Intrinsic, Scalable and Electrically-tunable Majorana Platform," arXiv:1905.10648.

[38] Y. You, D. Litinski, and F. von Oppen, "Higher-order topological superconductors as generators of quantum codes," Phys. Rev. B 100, 054513 (2019).

[39] R. W. Bomantara and J. Gong, "Measurement-only quantum computation with Floquet Majorana corner modes," Phys. Rev. B 101, 085401 (2020).

[40] S.-B. Zhang, W. B. Rui, A. Calzona, S.-J. Choi, A. P. Schnyder, and B. Trauzettel, "Topological and holonomic quantum computation based on second-order topological superconductors," arXiv:2002.05741.

[41] X. Zhu, "Tunable Majorana corner states in a twodimensional second-order topological superconductor induced by magnetic fields," Phys. Rev. B 97, 205134 (2018).

[42] M. Ezawa, "Braiding of Majorana-like corner states in electric circuits and its non-Hermitian generalization," Phys. Rev. B 100, 045407 (2019).

[43] T. E. Pahomi, M. Sigrist, and A. A. Soluyanov, "Braiding Majorana corner modes in a two-layer second-order topological insulator," arXiv:1904.07822.

[44] S. Bravyi, "Universal quantum computation with the $\nu=$ 5/2 fractional quantum Hall state," Phys. Rev. A 73, 042313 (2006).

[45] We note, however, that our main results discussed below also apply to other cases, for instance, AFM with $H_{M}=$ $M_{0} \tau_{z} s_{x} \sigma_{x}$.

[46] See the Supplemental Material for details, which includes Refs. [48-50, 70].

[47] L. Fu and C. L. Kane, "Superconducting Proximity Effect and Majorana Fermions at the Surface of a Topological Insulator," Phys. Rev. Lett. 100, 096407 (2008).

[48] J. Alicea, Y. Oreg, G. Refael, F. Von Oppen, and M. P. A. Fisher, "Non-Abelian statistics and topological quantum information processing in 1D wire networks," Nat. Phys. 7, 412 (2011).

[49] K. Flensberg, "Non-Abelian Operations on Majorana Fermions via Single-Charge Control," Phys. Rev. Lett. 106, 090503 (2011).

[50] X. Wu, S. Qin, Y. Liang, H. Fan, and J. Hu, "Topological characters in $\mathrm{Fe}\left(\mathrm{Te}_{1-x} \mathrm{Se}_{x}\right)$ thin films," Phys. Rev. B 93, 115129 (2016).

[51] X. Shi, Z.-Q. Han, P. Richard, X.-X. Wu, X.-L. Peng, T. Qian, S.-C. Wang, J.-P. Hu, Y.J. Sun, and H. Ding, "FeTe ${ }_{1-x} \mathrm{Se}_{x}$ monolayer films: towards the realization of high-temperature connate topological superconductivity," Science bulletin 62, 503-507 (2017).

[52] X.-L. Peng, Y. Li, X.-X. Wu, H.-B. Deng, X. Shi, W.-H. Fan, M. Li, Y.-B. Huang, T. Qian, P. Richard, J.-P. Hu, S.-H. Pan, H.-Q. Mao, Y.-J. Sun, and H. Ding, "Observation of topological transition in high- $T_{c}$ superconducting monolayer $\mathrm{FeTe}_{1-x} \mathrm{Se}_{x}$ films on $\mathrm{SrTiO}_{3}(001)$," Phys. Rev. B 100, 155134 (2019).

[53] F. Li, H. Ding, C. Tang, J. Peng, Q. Zhang, W. Zhang, G. Zhou, D. Zhang, C.-L. Song, K. He, S. Ji, X. Chen, L. Gu, L. Wang, X.-C. Ma, and Q.-K. Xue, "Interface- enhanced high-temperature superconductivity in singleunit-cell $\mathrm{FeTe}_{1-x} \mathrm{Se}_{x}$ films on $\mathrm{SrTio}_{3}$," Phys. Rev. B 91, 220503 (2015).

[54] The pairing potential in $\mathrm{FeTe}_{1-x} \mathrm{Se}_{x}$ may be $s_{ \pm}$-wave. In this case, we should replace $\Delta_{0} \rightarrow \Delta_{0}+\Delta_{2}\left(\cos k_{x}+\right.$ $\left.\cos k_{y}\right)$ in the bulk Hamiltonian. The momentum correction in the pairing potential modifies the effective pairing potential on the boundary to $\Delta_{0} \rightarrow \Delta_{0}+\Delta_{2} m_{0} / m$ and thus does not change the results qualitatively.

[55] F. Ma, W. Ji, J. Hu, Z.-Y. Lu, and T. Xiang, "FirstPrinciples Calculations of the Electronic Structure of Tetragonal $\alpha$-FeTe and $\alpha$-FeSe Crystals: Evidence for a Bicollinear Antiferromagnetic Order," Phys. Rev. Lett. 102, 177003 (2009).

[56] W. Bao, Y. Qiu, Q. Huang, M. A. Green, P. Zajdel, M. R. Fitzsimmons, M. Zhernenkov, S. Chang, M. Fang, B. Qian, E. K. Vehstedt, J. Yang, H. M. Pham, L. Spinu, and Z. Q. Mao, "Tunable $(\delta \pi, \delta \pi)$-Type Antiferromagnetic Order in $\alpha$-Fe(Te,Se) Superconductors," Phys. Rev. Lett. 102, 247001 (2009).

[57] S. Manna, A. Kamlapure, L. Cornils, T. Hänke, E. M. J. Hedegaard, M. Bremholm, B. B. Iversen, P. Hofmann, J. Wiebe, and R. Wiesendanger, "Interfacial superconductivity in a bi-collinear antiferromagnetically ordered FeTe monolayer on a topological insulator," Nat. Commun. 8, 14074 (2017).

[58] M. B Salamon, N. Cornell, M. Jaime, F. F. Balakirev, A. Zakhidov, J. Huang, and H. Wang, "Upper Critical Field and Kondo Effects in $\mathrm{Fe}\left(\mathrm{Te}_{0.9} \mathrm{Se}_{0.1}\right)$ Thin Films by Pulsed Field Measurements," Sci. Rep. 6, 21469 (2016).

[59] N. Hao and J. Hu, "Topological Phases in the SingleLayer FeSe," Phys. Rev. X 4, 031053 (2014).

[60] N. Hao and J. Hu, "Topological quantum states of matter in iron-based superconductors: from concept to material realization," Natl. Sci. Rev. 6, 213-226 (2018).

[61] Z. F. Wang, H. Zhang, D. Liu, C. Liu, C. Tang, C. Song, Y. Zhong, J. Peng, F. Li, C. Nie, et al., "Topological edge states in a high-temperature superconductor FeSe/SrTiO 3 (001) film," Nat. Mater. 15, 968-973 (2016).

[62] S. He, J. He, W. Zhang, L. Zhao, D. Liu, X. Liu, D. Mou, Y.-B. Ou, Q.-Y. Wang, Z. Li, et al., "Phase diagram and electronic indication of high-temperature superconductivity at $65 \mathrm{~K}$ in single-layer FeSe films," Nat. Mater. 12, 605-610 (2013).

[63] X. Qian, J. Liu, L. Fu, and J. Li, "Quantum spin Hall effect in two-dimensional transition metal dichalcogenides," Science 346, 1344 (2014).

[64] S. Wu, V. Fatemi, Q. D. Gibson, K. Watanabe, T. Taniguchi, R. J. Cava, and P. Jarillo-Herrero, "Observation of the quantum spin Hall effect up to 100 kelvin in a monolayer crystal," Science 359, 76 (2018).

[65] Z. Fei, T. Palomaki, S. Wu, W. Zhao, X. Cai, B. Sun, P Nguyen, J. Finney, X. Xu, and D. H. Cobden, "Edge conduction in monolayer WTe 2 ," Nat. Phys. 13, 677-682 (2017).

[66] S. Tang, C. Zhang, D. Wong, Z. Pedramrazi, H.-Z. Tsai, C. Jia, B. Moritz, M. Claassen, H. Ryu, S. Kahn, et al., "Quantum spin Hall state in monolayer 1T'-WTe 2 ," Nat. Phys. 13, 683 (2017).

[67] E. Sajadi, T. Palomaki, Z. Fei, W. Zhao, P. Bement, C. Olsen, S Luescher, X. Xu, J. A. Folk, and D. H. Cobden, "Gate-induced superconductivity in a monolayer topological insulator," Science 362, 922-925 (2018).

[68] V. Fatemi, S. Wu, Y. Cao, L. Bretheau, Q. D. Gibson, 
K. Watanabe, T. Taniguchi, R. J. Cava, and P. JarilloHerrero, "Electrically tunable low-density superconductivity in a monolayer topological insulator," Science $\mathbf{3 6 2}$, 926-929 (2018).

[69] F. Lüpke, D. Waters, C. Sergio, M. Widom, D. G. Mandrus, J. Yan, R. M. Feenstra, and B. M. Hunt, "Proximity-induced superconducting gap in the quantum spin Hall edge state of monolayer WTe 2 ," Nat. Phys. 16, 526-530 (2020).

[70] B. A. Bernevig, T. L. Hughes, and S.-C. Zhang, "Quantum Spin Hall Effect and Topological Phase Transition in HgTe Quantum Wells," Science 314, 1757 (2006).

[71] M. König, S. Wiedmann, C. Brüne, A. Roth, H. Buhmann, L. W. Molenkamp, X.-L. Qi, and S.-C. Zhang, "Quantum spin Hall insulator state in $\mathrm{HgTe}$ quantum wells," Science 318, 766 (2007).

[72] C. Liu, T. L. Hughes, X.-L. Qi, K. Wang, and S.-C. Zhang, "Quantum Spin Hall Effect in Inverted Type-II Semiconductors," Phys. Rev. Lett. 100, 236601 (2008).

[73] I. Knez, R. R. Du, and G. Sullivan, "Evidence for Helical Edge Modes in Inverted InAs/GaSb Quantum Wells," Phys. Rev. Lett. 107, 136603 (2011).

[74] S. Hart, H. Ren, T. Wagner, P. Leubner, M. Mühlbauer, C. Brüne, H. Buhmann, L. W. Molenkamp, and A. Yacoby, "Induced superconductivity in the quantum spin Hall edge," Nat. Phys. 10, 638 (2014).

[75] S. Hart, H. Ren, M. Kosowsky, G. Ben-Shach, P. Leubner, C. Brüne, H. Buhmann, L. W. Molenkamp, B. I. Halperin, and A. Yacoby, "Controlled finite momentum pairing and spatially varying order parameter in proximitized HgTe quantum wells," Nat. Phys. 13, 87-93 (2017).

[76] H. Ren, F. Pientka, S. Hart, A. T. Pierce, M. Kosowsky, L. Lunczer, R. Schlereth, B. Scharf, E. M Hankiewicz, L. W. Molenkamp et al., "Topological superconductivity in a phase-controlled Josephson junction," Nature (London) 569, 93 (2019).

[77] A.M. Goldman, "Electrostatic gating of ultrathin films," Ann. Rev. Mater. Res. 44, 45-63 (2014).

[78] K. Hanzawa, H. Sato, H. Hiramatsu, T. Kamiya, and H. Hosono, "Electric field-induced superconducting transition of insulating FeSe thin film at 35 K," Proc. Natl. Acad. Sci. U. S. A. 113, 3986-3990 (2016).

[79] J. Hänisch, K. Iida, R. Hüne, and C. Tarantini, "Fe-based superconducting thin films-preparation and tuning of superconducting properties," Supercond. Sci. Tech. 32, 093001 (2019).

[80] G. Goldstein and C. Chamon, "Decay rates for topological memories encoded with Majorana fermions," Phys. Rev. B 84, 205109 (2011).

[81] J. C. Budich, S. Walter, and B. Trauzettel, "Failure of protection of Majorana based qubits against decoherence," Phys. Rev. B 85, 121405 (2012).

[82] D. Rainis and D. Loss, "Majorana qubit decoherence by quasiparticle poisoning," Phys. Rev. B 85, 174533 (2012).

[83] G. C. Ménard, F. K. Malinowski, D. Puglia, D. I. Pikulin, T. Karzig, B. Bauer, P. Krogstrup, and C. M. Marcus, "Suppressing quasiparticle poisoning with a voltagecontrolled filter," Phys. Rev. B 100, 165307 (2019). 


\section{Supplemental Material}

In this Supplemental Material, we derive the low-energy effective boundary Hamiltonian and the wavefunctions of Majorana bound states (MBSs) of the second-order topological superconductors (SOSTs) in Sec. A. In Sec. B, we analyze the motion and the localization properties of MBSs on a single triangle island when varying the chemical potential, the excitation energy gap during the Majorana motion, the influence of adding small bending to the triangle diagonals, and provide numerical simulations of MBS pairs on the network. We discuss the measurement of Majorana qubits via quantum dots in Sec. C. We numerically study the robustness of our results with respect to moderate inhomogeneities of chemical potential within individual triangle islands in Sec. D. Finally, in Sec. E, we discuss alternative examples of Majorana networks.

\section{Appendix A: Derivations of the effective boundary Hamiltonian and wavefunctions of Majorana bound states}

\section{Effective boundary Hamiltonian}

In this subsection, we derive the effective boundary Hamiltonian on a disk geometry. To do so, we first derive the boundary states in the absence of magnetic and superconducting order. The low-energy Hamiltonian without magnetic and superconducting order decouples into four blocks which are respectively for spin-up, spindown electrons, spin-up, and spin-down holes. These four blocks are related by time-reversal and particlehole symmetries. In the following, we take the block for spin-up electrons for illustration. In polar coordinates, $r=\sqrt{x^{2}+y^{2}}$ and $\varphi=\arctan (y / x)$, this block Hamiltonian is given by

$$
h_{e \uparrow}=\left(\begin{array}{cc}
m\left(\partial^{2}\right) & -A e^{-i \varphi}\left(i \partial_{r}+r^{-1} \partial_{\varphi}\right) \\
-A e^{i \varphi}\left(i \partial_{r}-r^{-1} \partial_{\varphi}\right) & -m\left(\partial^{2}\right)
\end{array}\right) \text {, }
$$

where $m\left(\partial^{2}\right)=m_{0}+m\left(\partial_{r}^{2}+r^{-2} \partial_{\varphi}^{2}+r^{-1} \partial_{r}\right)$. In the disk without magnetic order, the angular momentum $\nu$ is a good quantum number. Consider large radius $R \gg$ $|m / A|$. We can assume an ansatz for the boundary-state wavefunction as

$$
\psi(\mathbf{x})=\left(e^{i \nu \varphi} e^{\lambda r} / \sqrt{r}\right)\left(\alpha, \beta e^{i \varphi}\right)^{T},
$$

where $\mathbf{x} \equiv(r, \varphi)$. The $\varphi$ periodicity of the wavefunction $\psi(r, \varphi)=\psi(r, \varphi+2 \pi)$ imposes the constraint $\nu \in \mathbb{Z} \equiv$ $\{0, \pm 1, \pm 2, \cdots\}$. Plugging the ansatz (A2) into the Dirac equation for a given energy $\epsilon$, and solving the equation, we find four solutions of $\lambda$ as $\pm \lambda_{1 / 2}$, where

$$
\begin{aligned}
\lambda_{1 / 2}^{2}= & (\nu+1 / 2)^{2} / r^{2}-\left(2 m m_{0}-v^{2}\right) /\left(2 m^{2}\right) \\
& \pm\left(A^{4}-4 m m_{0} A^{2}-4 m^{2} \epsilon_{\nu}^{2}\right)^{1 / 2} /\left(2 m^{2}\right),
\end{aligned}
$$

and correspondingly four solutions of $(\alpha, \beta)^{T}$, where $\epsilon_{\nu}=$ $\epsilon-m(\nu+1 / 2) / r^{2}$. The boundary states are localized on the boundary. We thus expand the wavefunctions as

$$
\Psi_{e, \uparrow}(\mathbf{x})=\sum_{j=1,2} C_{\lambda_{j}} e^{i \nu \varphi} \frac{e^{\lambda_{j} r}}{\sqrt{r}}\left(\begin{array}{c}
i A\left[\lambda_{j}+(\nu+1 / 2) / r\right] \\
\left(m_{\nu}+m \lambda_{j}^{2}-\epsilon_{\nu}\right) e^{i \varphi}
\end{array}\right),
$$

where $m_{\nu}=m_{0}-m(\nu+1 / 2)^{2} / r^{2}$ and $\operatorname{Re}\left[\lambda_{1 / 2}(R)\right]>0$ have been assumed without loss of generality. Imposing open boundary conditions to this wavefunction

$$
\Psi_{e, \uparrow}(r=R, \varphi)=0
$$

the allowed energy of boundary states can be found explicitly as

$$
\epsilon(\nu)=-\operatorname{sgn}(m)|A| \nu / R+m \nu / R^{2} .
$$

Hence, the coefficients $C_{\lambda_{1}}$ and $C_{\lambda_{2}}$ are also found. The resulting wavefunctions can be written as

$$
\Psi_{e, \uparrow}(\mathbf{x})=e^{i \nu \varphi} K(r)\left(\operatorname{sgn}(m A),-i e^{i \varphi}\right)^{T},
$$

where $K(r)=\mathcal{N}\left[e^{\lambda_{1}(r-R)}-e^{\lambda_{2}(r-R)}\right] / \sqrt{r}, \lambda_{1 / 2}=$ $|A /(2 m)| \pm\left(A^{2} /\left(4 m^{2}\right)-m_{0} / m+(\nu+1 / 2)^{2} / R^{2}\right)^{1 / 2}$ and $\mathcal{N}$ is the normalization factor.

For large $R \gg|m / A|$, we approximate a small segment of the disk boundary as a straight line. Define an effective coordinate and corresponding momentum as

$$
s \equiv R \varphi, \quad p_{\varphi} \equiv \nu / R .
$$

Then, the dispersion relation (A6) becomes

$$
E_{e, \uparrow}\left(p_{\varphi}\right)=-\operatorname{sgn}(m)|A| p_{\varphi}-\mu,
$$

and the corresponding wavefunction (A7)

$$
\Psi_{e, \uparrow, p_{\varphi}}(\mathbf{x})=e^{i p_{\varphi} s} K(r)\left(\operatorname{sgn}(m A),-i e^{i \varphi}\right)^{T},
$$

where $\lambda_{1,2}=|A /(2 m)| \pm\left(A^{2} /\left(4 m^{2}\right)-m_{0} / m+p_{\varphi}^{2}\right)^{1 / 2}$ and $K(r)=\mathcal{N}\left[e^{\lambda_{1}(r-R)}-e^{\lambda_{2}(r-R)}\right] / \sqrt{R}$. In Eq. (A9), we have considered the chemical potential $\mu$. In the full basis of the bulk Hamiltonian, the wavefunction reads

$$
\Psi_{e, \uparrow, p_{\varphi}}(\mathbf{x})=e^{i p_{\varphi} s} K(r)\left(\operatorname{sgn}(m A),-i e^{i \varphi}, 0,0,0,0,0,0\right)^{T} .
$$

Exploiting time-reversal and particle-hole symmetries, we obtain easily the results for spin-down electrons, spinup and spin-down holes as

$$
\begin{aligned}
& E_{e, \downarrow}\left(p_{\varphi}\right)=E_{e, \downarrow}\left(-p_{\varphi}\right), \\
& E_{h, \uparrow}\left(p_{\varphi}\right)=-E_{e, \downarrow}\left(-p_{\varphi}\right), \\
& E_{h, \downarrow}\left(p_{\varphi}\right)=-E_{e, \uparrow}\left(-p_{\varphi}\right),
\end{aligned}
$$


and

$$
\begin{aligned}
\Psi_{e, \downarrow, p_{\varphi}}(\mathbf{x}) & =i s_{y} \Psi_{e, \uparrow,-p_{\varphi}}^{*}(\mathbf{x}), \\
\Psi_{h, \uparrow / \downarrow, p_{\varphi}}(\mathbf{x}) & =\tau_{x} \Psi_{e, \uparrow / \downarrow,-p_{\varphi}}^{*}(\mathbf{x}) .
\end{aligned}
$$

These boundary states are helical with velocity $A$ : the spin-up electrons move in one direction and the spindown electrons move in the opposite direction.

Using the helical states $\left(\Psi_{e, \uparrow}, \Psi_{e, \downarrow}, \Psi_{h, \uparrow}, \Psi_{h, \downarrow}\right)$ as basis, we now project the superconducting and magnetic order onto it. The projection can be performed as

$$
\widetilde{\mathcal{H}}_{i, j}=\left\langle\Psi_{i}\left|\left(\Delta_{0} \tau_{y} s_{y}+H_{M}\right)\right| \Psi_{j}\right\rangle,
$$

where the subscript $i, j$ are abbreviations of the indices $(e / h, \uparrow / \downarrow)$. Assuming $m A>0$ without loss of generality, we obtain the effective boundary Hamiltonian stated in Eq. (2) of the main text.

\section{Wavefunctions of Majorana bound states}

Next, we derive the wavefunctions of MBSs. Assume the wavefunction for the MBS $\gamma_{i}$ around its localization center $\varphi_{i}$ in the form

$$
\Psi_{0}=e^{R \int \xi(\varphi) d \varphi}\left(c_{1}, c_{2}, c_{3}, c_{4}\right)^{T} .
$$

The eigen equation at zero energy is then given by where $e^{i \vartheta}=\left(\Delta_{0}+i \mu\right) / \bar{\Delta}$. Considering the continuity of the wavefunction at $\varphi=\varphi_{1}$, we find $\alpha_{>}=\alpha_{<}=0$ and $\beta_{>}=\beta_{<}$. Therefore, $\Psi_{1}$ can be simplified to

$$
\Psi_{1}=e^{\int \xi_{2} R d \varphi}\left(-i,-i e^{-i \vartheta} e^{i \varphi_{1}}, e^{-i \vartheta} e^{i \varphi_{1}}, 1\right)^{T} .
$$

In the original basis $\left(c_{a \uparrow}, c_{b \uparrow}, c_{a \downarrow}, c_{b \downarrow}, c_{a \uparrow}^{\dagger}, c_{b \uparrow}^{\dagger}, c_{a \downarrow}^{\dagger}, c_{b \downarrow}^{\dagger}\right)$, the wavefunction can be written as

$$
\begin{aligned}
\Psi_{1}=e^{\int \xi_{2} R d \varphi} & K(r)\left(e^{-i\left(\varphi_{1}-\vartheta+\pi / 2\right) / 2},-e^{i\left(\varphi_{1}+\vartheta+\pi / 2\right) / 2},\right. \\
& e^{i\left(\varphi_{1}-\vartheta-\pi / 2\right) / 2}, e^{-i\left(\varphi_{1}+\vartheta-\pi / 2\right) / 2}, \\
& e^{i\left(\varphi_{1}-\vartheta+\pi / 2\right) / 2},-e^{-i\left(\varphi_{1}+\vartheta+\pi / 2\right) / 2}, \\
& \left.e^{-i\left(\varphi_{1}-\vartheta-\pi / 2\right) / 2}, e^{i\left(\varphi_{1}+\vartheta-\pi / 2\right) / 2}\right)^{T},(\mathrm{~A} 21)
\end{aligned}
$$

up to a phase factor.

Similarly, we can derive the wavefunctions of the other three MBSs as

$$
\begin{aligned}
\Psi_{2}=e^{-\int \xi_{2} R d \varphi} & K(r)\left(-e^{-i\left(\varphi_{2}+\vartheta+\pi / 2\right) / 2}, e^{i\left(\varphi_{2}-\vartheta+\pi / 2\right) / 2},\right. \\
& e^{i\left(\varphi_{2}+\vartheta-\pi / 2\right) / 2}, e^{-i\left(\varphi_{2}-\vartheta-\pi / 2\right) / 2}, \\
& -e^{i\left(\varphi_{2}+\vartheta+\pi / 2\right) / 2}, e^{-i\left(\varphi_{2}-\vartheta+\pi / 2\right) / 2}, \\
& \left.e^{-i\left(\varphi_{2}+\vartheta-\pi / 2\right) / 2}, e^{i\left(\varphi_{2}-\vartheta-\pi / 2\right) / 2}\right)^{T}, \\
\Psi_{3}=e^{\int \xi_{2} R d \varphi} & K(r)\left(e^{-i\left(\varphi_{3}-\vartheta-\pi / 2\right) / 2}, e^{i\left(\varphi_{3}+\vartheta-\pi / 2\right) / 2},\right. \\
& -e^{i\left(\varphi_{3}-\vartheta+\pi / 2\right) / 2}, e^{-i\left(\varphi_{3}+\vartheta+\pi / 2\right) / 2}, \\
& e^{i\left(\varphi_{3}-\vartheta-\pi / 2\right) / 2}, e^{-i\left(\varphi_{3}+\vartheta-\pi / 2\right) / 2}, \\
& \left.-e^{-i\left(\varphi_{3}-\vartheta+\pi / 2\right) / 2}, e^{i\left(\varphi_{3}+\vartheta+\pi / 2\right) / 2}\right)^{T},
\end{aligned}
$$

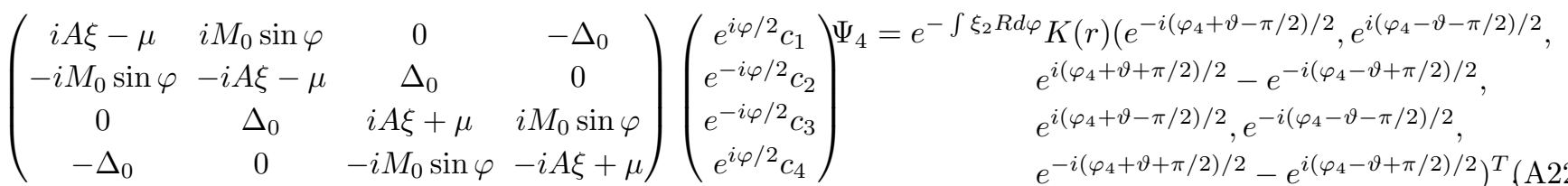

$=0$.

We can observe from these wavefunctions that $\Psi_{3}=$ $-\mathcal{P} \Psi_{1}$ and $\Psi_{4}=\mathcal{P} \Psi_{2}$, where $\mathcal{P}=\sigma_{z} \mathcal{T}_{\varphi \rightarrow \varphi+\pi}$ is the inversion symmetry operator and $\mathcal{T}_{\varphi \rightarrow \varphi+\pi}=e^{-i \pi \tau_{z} s_{z} \sigma_{z} / 2}$ shifts the angle $\varphi$ by $\pi$. This indicates that the modes $\gamma_{1}$ and $\gamma_{2}$ are inversion partners of $\gamma_{3}$ and $\gamma_{4}$, respectively. Therefore, in the SOTS with inversion symmetry in the bulk, the scattering between $\gamma_{1}$ and $\gamma_{3}$ (or $\gamma_{2}$ and $\gamma_{4}$ ) is prohibited, as verified by $F_{\gamma_{1}: \gamma_{3}}=F_{\gamma_{2}: \gamma_{4}}=0$.

The function $\xi_{2}(\varphi)$ in the wavefunctions determines the localization length of the MBSs along the boundary. It can equivalently determine the localization length of the MBSs on a straight boundary which is normal to the azimuthal direction at $\varphi$. According to Eq. (A17), the localization length on this straight boundary is approximately given by

$\Psi_{1}=\left\{\begin{array}{l}\alpha_{>} e^{-\int \xi_{1} R d \varphi}\left(i, i e^{i \vartheta} e^{i \varphi_{1}}, e^{i \vartheta} e^{i \varphi_{1}}, 1\right)^{T} \\ +\beta_{>} e^{\int \xi_{2} R d \varphi}\left(-i,-i e^{-i \vartheta} e^{i \varphi_{1}}, e^{-i \vartheta} e^{i \varphi_{1}}, 1\right)^{T}, \quad \varphi>\varphi_{1} \\ \alpha_{<} e^{\int \xi_{1} R d \varphi}\left(-i, i e^{-i \vartheta} e^{i \varphi_{1}},-e^{-i \vartheta} e^{i \varphi_{1}}, 1\right)^{T} \\ +\beta_{<} e^{\int \xi_{2} R d \varphi}\left(-i,-i e^{-i \vartheta} e^{i \varphi_{1}}, e^{-i \vartheta} e^{i \varphi_{1}}, 1\right)^{T}, \quad \varphi<\varphi_{1}\end{array}\right.$

$l_{\text {decay }} \approx A \Delta_{0} /\left(\mu_{\varphi}\left|\mu-\mu_{\varphi}\right|\right)$, $\mu$ approaches the critical value $\mu_{\varphi}$. 


\section{Appendix B: Numerical simulations of moving Majorana bound states}

\section{MBSs in a single isosceles right triangle island}

In this subsection, we study numerically the motion of MBSs in a single isosceles right triangle island (IRTI). We discretize the bulk Hamiltonian onto a tight-binding lattice (by replacing $k_{i} \rightarrow \sin \left(k_{i} a\right) / a$ and $k_{i}^{2} \rightarrow 2[1-$ $\left.\left.\cos \left(k_{i} a\right)\right], i \in\{x, y\}\right)$ and consider the short-side length of the triangle as $L=50 a$ for concreteness. Several subsequent snapshots of the positions of the two MBSs in an IRTI when increasing the chemical potential are displayed in Fig. S5. Other parameters are given in the caption. The corresponding animation that shows the slow movement of MBSs is provided in the supplemental file "Triangle-movie.mp4". In this simulation, we see clearly that one MBS is moved slowly from one sharp-angle vertex to the other one along the diagonal while the other MBS is kept untouched at the right-angle vertex. This behavior is of perfect consistency with our analytical conclusion.

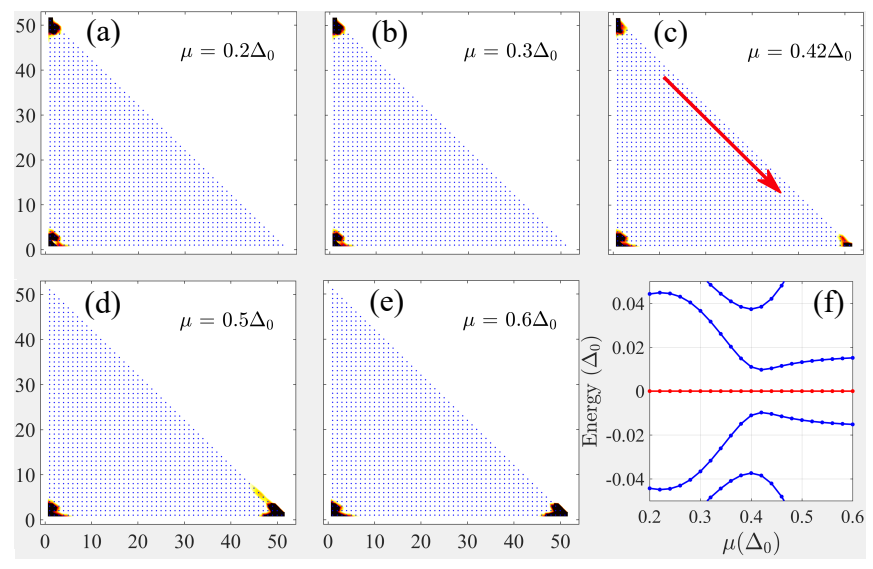

Fig. S5. (a)-(e) Several subsequent snapshots show the positions (the black densities) of the MBSs in an IRTI when increasing $\mu$ across $\mu_{c}$. (f) Energy spectrum of this process. At the critical chemical potential $\mu_{c}$, the MBS on the diagonal is partially localized at one sharp-angle vertex and partially at the other sharp-angle vertex. The parameters are $\Delta_{0}=0.25 m_{0}, M_{0}=0.4 m_{0}, A=m=0.5 m_{0}=1$, and the short-side length of the IRTI is $L=50 a$ with $a$ the lattice constant.

Figure S6 shows the logarithm of the probability density $\left[\log \left(\left|\Psi_{i}\right|^{2}\right)\right]$ of the movable Majorana wavefunction along the diagonal for different chemical potentials $\mu$, where $i$ labels the $y$ coordinate of the lattice sites. The lines are linear fittings on the sites between $10<i<40$. In the central part of the diagonal, the decay of the wavefunction is clearly exponential, as expected. By increasing $\mu$, we can observe that the MBS localizing at the left edge $(i=50)$ moves to localize at the right one $(i=0)$. The localization length of the MBS wavefunc-
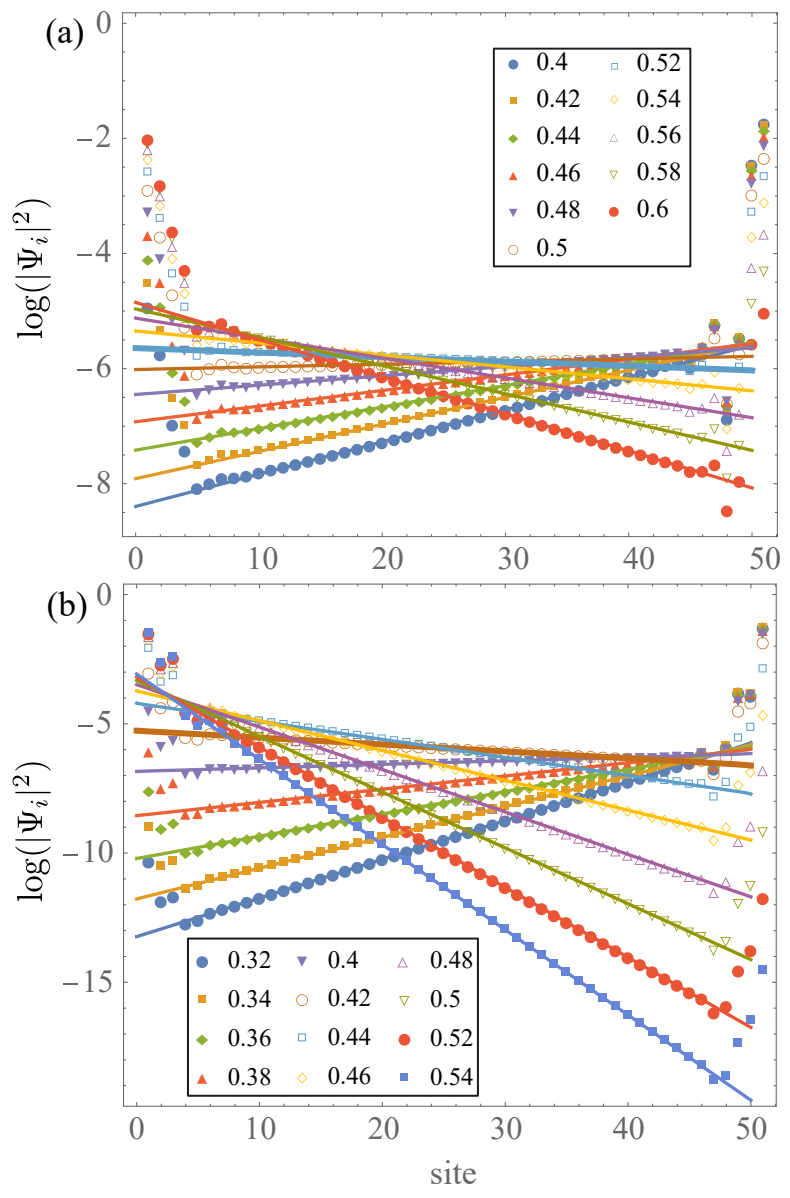

Fig. S6. Logarithm of the probability density of the movable Majorana wavefunction along the diagonal of an IRTI for different chemical potentials (indicated in the legend and in units of $\left.\Delta_{0}\right)$. The colored dots are numerical data and the lines are linear fittings on the sites between $10<i<40$. The value of $\mu$ corresponding to the flat fitting line gives the critical chemical potential $\bar{\mu}_{c}$. When $\Delta_{0}$ and $M_{0}$ are much smaller than the insulating gap of the bulk $\left(\sim m_{0}\right), \bar{\mu}_{c}$ agrees excellently with the analytical result $\mu_{c}\left(=0.53 \Delta_{0}\right)$, see the curve of $\mu=0.52 \Delta_{0}$ (a). In contrast, when $\Delta_{0}$ and $M_{0}$ become comparable with $m_{0}, \bar{\mu}_{c}$ deviates evidently from $\mu_{c}$, see the curve of $\mu=0.42 \Delta_{0}$ in (b). $\Delta_{0}=0.1 m_{0}$ for (a) and $\Delta_{0}=0.25 m_{0}$ for (b), other parameters are $M_{0}=1.6 \Delta_{0}, A=m=0.5 m_{0}$ and the short-side length of the IRTI is $L=50 a$.

tion can be extracted as the inverse of the slopes of the fitting lines. It diverges at $\bar{\mu}_{c}$ which corresponds to the critical value of chemical potential. When $\Delta_{0}$ and $M_{0}$ are small compared to $m_{0}, \bar{\mu}_{c}$ agrees excellently with its analytical value given by $\mu_{c}=\sqrt{M_{0}^{2} / 2-\Delta_{0}^{2}}$, see Fig. S6(a). In contrast, when $\Delta_{0}$ and $M_{0}$ are comparable with $m_{0}$, the critical chemical potential becomes significantly smaller than $\mu_{c}$, see Fig. S6(b). This deviation could be attributed to higher-order momentum corrections in the tight-binding calculation.

At the critical chemical potential $\mu_{c}$, the energy gap is minimal. This may be related to the fact that at $\mu=\mu_{c}$, 
the 1D diagonal edge effectively realizes a topological phase transition. It becomes gapless if it is infinitely long. A finite length of the diagonal, however, gives rise to an energy gap $\Delta E$, which is larger than $\geq A \pi / \sqrt{2} L$, where $L$ is the short-side length of the IRTI. For inverted $\mathrm{Hg}(\mathrm{Cd}) \mathrm{Te}$ quantum wells, $A>0.3 \mathrm{eV} \cdot \mathrm{nm}[70]$ and for $\mathrm{FeTe}_{1-x} \mathrm{Se}_{x}, A>0.2 \mathrm{eV} \cdot \mathrm{nm}[50]$. Thus, the estimated energy gap due to the 1D finite-size confinement in these two candidate materials could be larger than $0.67 \mathrm{meV}(8$ $\mathrm{K})$ and $0.44 \mathrm{meV}(5 \mathrm{~K})$ for a length of $L=1 \mu \mathrm{m}$, respectively. In Fig. S7, we calculate numerically this minimal energy gap for increasing sizes of the triangle $L$. Interestingly, it shows an even less pronounced dependence on $L$ (compared to the $\propto 1 / L$ dependence).

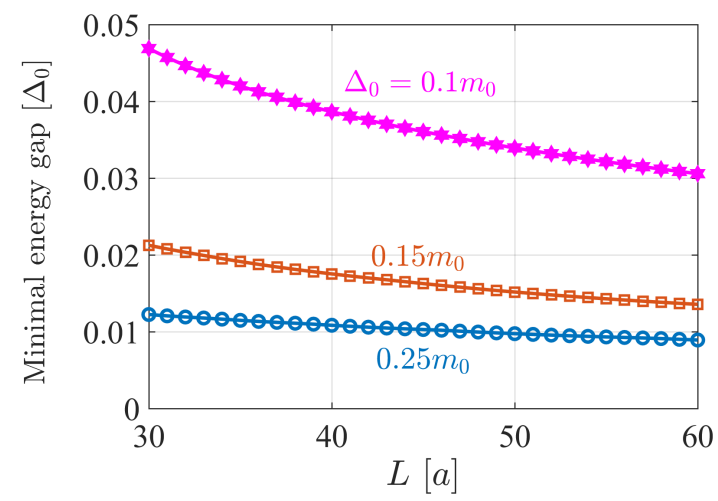

Fig. S7. The minimal energy gap $\Delta E$ as a function of the short-side length $L$ of the IRTI. The three curves correspond to three different pairing potentials, $\Delta_{0}=0.1 m_{0}, 0.15 m_{0}$ and $0.25 m_{0}$, respectively. $\Delta E$ decreases monotonically with increasing $L$ but at a rate less pronounced than $1 / L$. Other parameters for this numerical calculation are $M_{0}=1.6 \Delta_{0}$ and $A=m=0.5 m_{0}$.

\section{Effect of bending the diagonal}

In this section, we discuss the effect of small diagonal concavity and convexity bending on the IRTI, as sketched in Fig. S8. According to our effective boundary Hamiltonian, we can classify the boundary into two distinct classes: $\mathbb{A}$ with $\widetilde{M}>\bar{\Delta}$, and $\mathbb{B}$ with $\widetilde{M}<\bar{\Delta}$, where $\bar{\Delta}=\sqrt{\Delta_{0}^{2}+\mu^{2}}$ and $\widetilde{M}$ is the local effective magnetization. Then, an MBS forms and only forms at the domain that connects the boundaries of different classes.

In the case of concavity bending, the two short sides of the IRTI belong to classes $\mathbb{A}$ and $\mathbb{B}$, respectively. The diagonal arc, in general, is divided into two segments, one segment belongs to $\mathbb{A}$ and the other segment belongs to $\mathbb{B}$, as shown in Fig. S8(a). We have thus two MBSs, one stays fixed at the right-angle vertex and the other at the separating point on the diagonal. By tuning the chemical potential, we can move the separating point on the diagonal. Accordingly, one MBS moves along the di- (a)

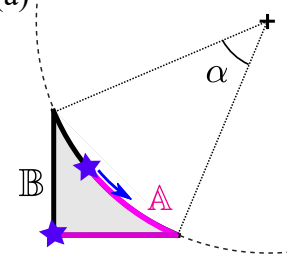

(b)

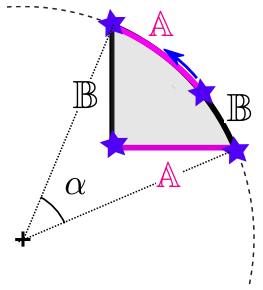

Fig. S8. Schematics of IRTIs with small concavity (a) or convexity (b) bending on the diagonal. The purple and black colors indicate that the boundary segments belong to classes $\mathbb{A}$ and $\mathbb{B}$, respectively. $\alpha$ denotes the angle of the diagonal arc. The pentagons represent the MBSs. The MBS on the diagonal arc can be smoothly moved by tuning $\mu$.

agonal. Note that the triangle diagonal behaves like a T-junction. Remarkably, only one single gate is needed for controlling the MBS, different from the T-junction of semiconducting nanowires which require many gates in a keyboard form [48]. These results are confirmed numerically in Fig. S9. It is important to note that the concavity bending on the diagonal can significantly enhance the energy gap that protects the zero-energy MBSs, compared to the case of straight diagonal, see Fig. S9(f). This enhancement can be attributed to the fact that the bent diagonal becomes gapped everywhere except at the separating point, even at the critical chemical potential.
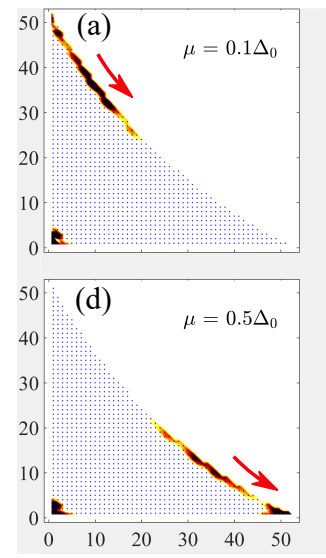
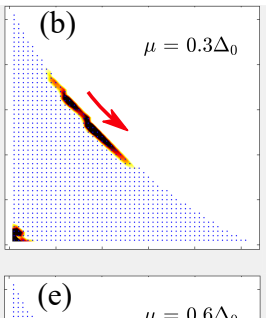

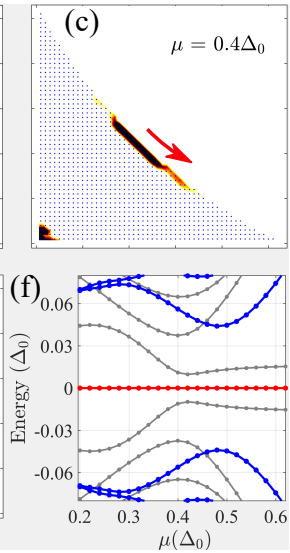

Fig. S9. (a)-(e) Several subsequent snapshots show the positions of the MBSs in an IRTI with a concavity bending of angle $\alpha=0.18 \pi$ in the diagonal. The corresponding chemical potentials are given in the panels. One MBS moves along the diagonal arc from one vertex to another vertex. (f) Energy spectrum (blue and red curves) of the process. The gray curves are for an IRTI without bending and are presented for comparison. The concavity bending in the diagonal significantly enhances the excitation gap that protects the MBSs in the system. Other parameters are the same as those in Fig. S5.

In the case of convexity bending, the diagonal arc is also divided into two segments but with exchanging their positions, as compared to the case of concavity bending. 

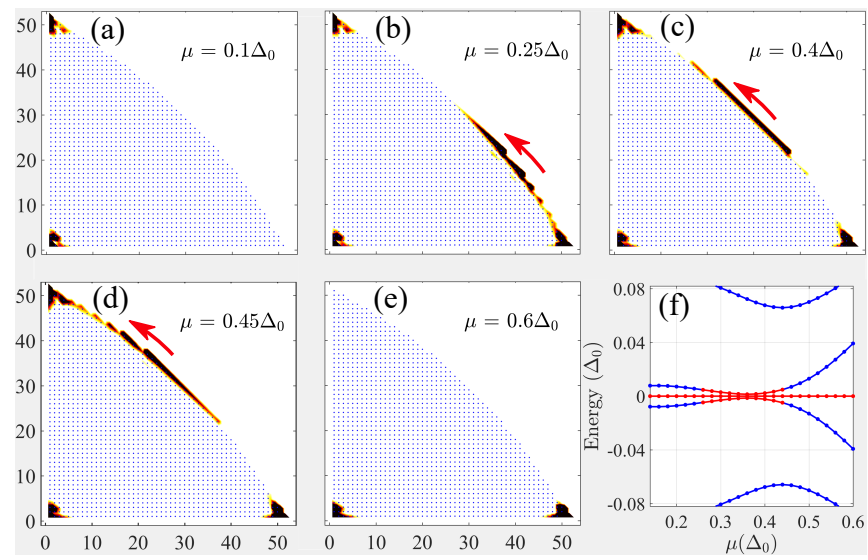

Fig. S10. (a)-(e) Several subsequent snapshots show the positions of the modes with energy lower than $0.005 \Delta_{0}$ in an IRTI with a convexity bending of angle $0.18 \pi$ in the diagonal. (f) Energy spectrum of the process. Increasing $\mu$, an extra pair of MBSs appear at the empty sharp-angle vertex. One of them moves along the diagonal arc to the other sharp-angle vertex and annihilates with the MBS there. Other parameters are the same as those in Fig. S5.

Thus, the triangle has four domains connecting the $\mathbb{A}$ and $\mathbb{B}$ boundaries and hence hosts four MBSs, see Fig. S8(b). Three of the four MBSs stay at the three vertices of the triangle, respectively, and the other on the diagonal arc. The latter is movable by adjusting the chemical potential. When it is close to another MBS at the sharpangle vertex, they annihilate together. We confirm these behaviors numerically in Fig. S10.

\section{Numerical simulations of exchanging MBS pairs in the illustrative network}

In Figs. S11 and S12, we present the numerical simulations of the exchanges of the MBS pairs, $\gamma_{b} \leftrightarrow \gamma_{c}$ and $\gamma_{c} \leftrightarrow \gamma_{d}$, respectively. These two exchanges, together with that of $\gamma_{a} \leftrightarrow \gamma_{b}$ (which is presented in the main text), generate the whole braid group of the four MBSs. For the exchange of $\gamma_{b} \leftrightarrow \gamma_{c}$, we turn the chemical potentials in the following successions: (i) $\mu_{5}=\mu_{\mathrm{u}} \rightarrow \mu_{\mathrm{d}}$; (ii) $\mu_{4}=\mu_{\mathrm{d}} \rightarrow \mu_{\mathrm{u}}$; (iii) $\mu_{3}=\mu_{\mathrm{d}} \rightarrow \mu_{\mathrm{u}}$; (iv) $\mu_{5}=\mu_{\mathrm{d}} \rightarrow \mu_{\mathrm{u}}$; (v) $\mu_{4}=\mu_{\mathrm{u}} \rightarrow \mu_{\mathrm{d}}$; and (vi) $\mu_{3}=\mu_{\mathrm{u}} \rightarrow \mu_{\mathrm{d}}$. For the exchange of $\gamma_{c} \leftrightarrow \gamma_{d}$, we tune (i) $\mu_{2}=\mu_{\mathrm{d}} \rightarrow \mu_{\mathrm{u}}$; (ii) $\mu_{4}=\mu_{\mathrm{d}} \rightarrow \mu_{\mathrm{u}}$; (iii) $\mu_{1}=\mu_{\mathrm{u}} \rightarrow \mu_{\mathrm{d}}$ and (iv) $\mu_{3}=\mu_{\mathrm{d}} \rightarrow \mu_{\mathrm{u}}$. The corresponding energy spectra are given in Figs. S11(h) and S12(h). The MBSs (red flat bands) are protected from excited modes (blue bands) by an excitation gap.
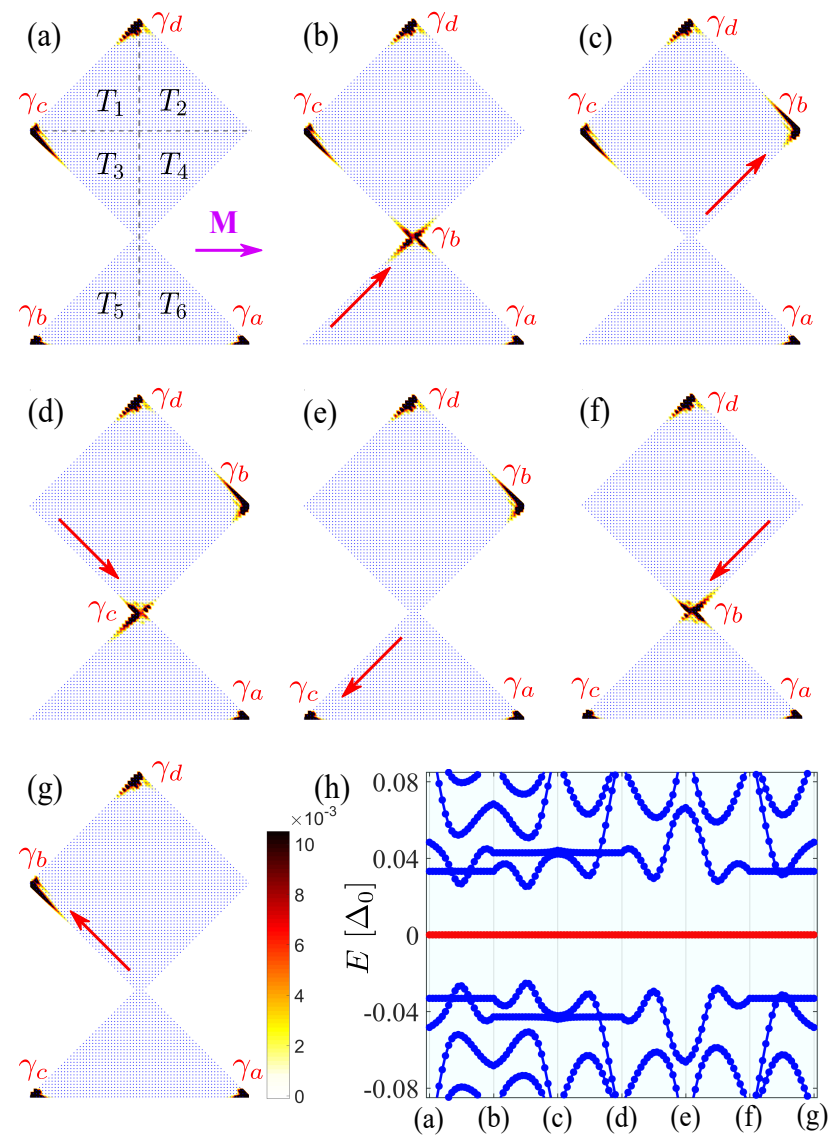

Fig. S11. Numerical simulation of the exchange of $\gamma_{b}$ and $\gamma_{c}$. Seven subsequent snapshots show the positions of MBSs at (a) $\boldsymbol{\mu}_{345} \equiv\left(\mu_{3}, \mu_{4}, \mu_{5}\right)=\left(\mu_{\mathrm{d}}, \mu_{\mathrm{d}}, \mu_{\mathrm{u}}\right)$, (b) $\boldsymbol{\mu}_{345}=$ $\left(\mu_{\mathrm{d}}, \mu_{\mathrm{d}}, \mu_{\mathrm{d}}\right),(\mathrm{c}) \boldsymbol{\mu}_{345}=\left(\mu_{\mathrm{d}}, \mu_{\mathrm{u}}, \mu_{\mathrm{d}}\right)$, (d) $\boldsymbol{\mu}_{345}=\left(\mu_{\mathrm{u}}, \mu_{\mathrm{u}}, \mu_{\mathrm{d}}\right)$, (e) $\boldsymbol{\mu}_{345}=\left(\mu_{\mathrm{u}}, \mu_{\mathrm{u}}, \mu_{\mathrm{u}}\right)$, (f) $\boldsymbol{\mu}_{345}=\left(\mu_{\mathrm{u}}, \mu_{\mathrm{d}}, \mu_{\mathrm{u}}\right)$, and $(\mathrm{g})$ $\boldsymbol{\mu}_{345}=\left(\mu_{\mathrm{d}}, \mu_{\mathrm{d}}, \mu_{\mathrm{u}}\right)$. (h) Energy spectrum of the system during the exchange process. $\mu_{1}=\mu_{6}=\mu_{\mathrm{u}}, \mu_{2}=\mu_{\mathrm{d}}$ and other parameters are the same as Fig. 3 of the main text.

\section{Appendix C: Measurement of Majorana qubits via quantum dots}

In this section, we briefly discuss the qubit measurement by using quantum dots. To do so, we turn off the connection at the junction (e.g., by applying an external gate which generates a large barrier potential) and move two measured MBSs, say again $\gamma_{b}$ and $\gamma_{c}$, to the two disconnected vertices, respectively, as sketched in Fig. S13(a). We assume a quantum dot (in red) nearby with a single energy level $\varepsilon$ and couple it elastically to $\gamma_{b}$ and $\gamma_{c}$ with coupling amplitudes $t_{b}$ and $t_{c}$, respectively. In the Coulomb blockade regime, the perturbed ground-state energy $E_{\text {tot }}$ of the dot depends on the total fermion parity of the dot and two MBSs [49], i.e., $E_{\text {tot }}=\varepsilon / 2-\left(\varepsilon^{2} / 4+\left|t_{b}^{2}\right|+\left|t_{c}^{2}\right|+2 \pm\left|t_{b} t_{c}\right| \sin \Phi_{b c}\right)^{1 / 2}$, where \pm corresponds to the fermion parity associated with the two MBSs $\gamma_{b}$ and $\gamma_{c}$, respectively, and $\Phi_{b c}=2 \operatorname{Arg}\left(t_{b} / t_{c}\right)$ 

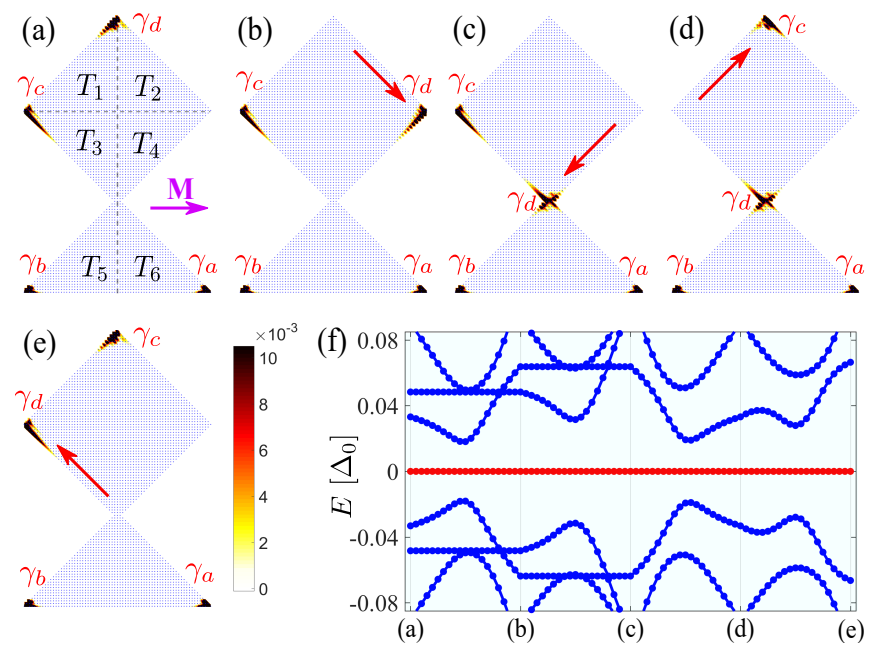

Fig. S12. Numerical simulation of the exchange of $\gamma_{c}$ and $\gamma_{d}$. Five subsequent snapshots show the positions of MBSs at (a) $\boldsymbol{\mu}_{1234} \equiv\left(\mu_{1}, \mu_{2}, \mu_{3}, \mu_{4}\right)=\left(\mu_{\mathrm{u}}, \mu_{\mathrm{d}}, \mu_{\mathrm{d}}, \mu_{\mathrm{d}}\right)$, (b) $\boldsymbol{\mu}_{1234}=$ $\left(\mu_{\mathrm{u}}, \mu_{\mathrm{u}}, \mu_{\mathrm{d}}, \mu_{\mathrm{d}}\right),(\mathrm{c}) \boldsymbol{\mu}_{1234}=\left(\mu_{\mathrm{u}}, \mu_{\mathrm{u}}, \mu_{\mathrm{d}}, \mu_{\mathrm{u}}\right),(\mathrm{d}) \boldsymbol{\mu}_{1234}=$ $\left(\mu_{\mathrm{d}}, \mu_{\mathrm{u}}, \mu_{\mathrm{d}}, \mu_{\mathrm{u}}\right)$, and (e) $\boldsymbol{\mu}_{1234}=\left(\mu_{\mathrm{d}}, \mu_{\mathrm{u}}, \mu_{\mathrm{u}}, \mu_{\mathrm{u}}\right)$. (f) Energy spectrum of the system during the process. $\mu_{5}=\mu_{6}=\mu_{\mathrm{u}}$ and other parameters are the same as Fig. 3 of the main text.

depends on $\Phi_{0}$. Suppose that the occupancy of the dot is known, then, the parity dependent energy $E_{\text {tot }}$ [see Fig. S13(b)] could provide an alternative method deduce the qubit states.
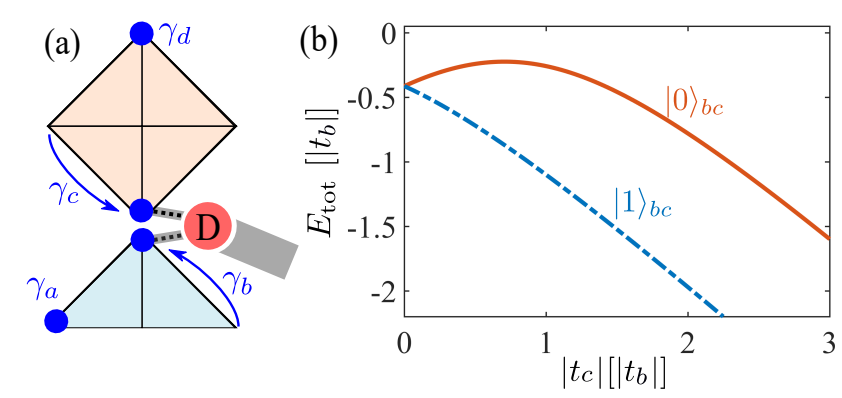

Fig. S13. (a) Schematics for probing the Majorana qubit via quantum dots. (b) Perturbed ground-state energy of the quantum dot coupled to the MBSs as a function of the coupling strength $\left|t_{c}\right|$. For concreteness, we assume that the single energy level $\varepsilon$ of the dot is empty. The solid and dashed curves correspond to the two qubit states $P_{b c}|0\rangle_{b c}=|0\rangle_{b c}$ and $P_{b c}\left|1_{b c}\right\rangle=-\left|1_{b c}\right\rangle . \varepsilon=3\left|t_{b}\right|$ and $\Phi_{b c}=\pi / 2$ are used for the plotting.

\section{Appendix D: robustness with respect to chemical potential inhomogeneities within individual IRTIs}

In all above simulations, we assume that the chemical potential is homogeneous in each individual IRTI. In realistic situations, however, variations of chemical po-

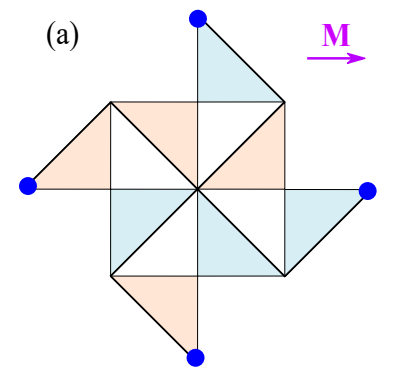

(b)
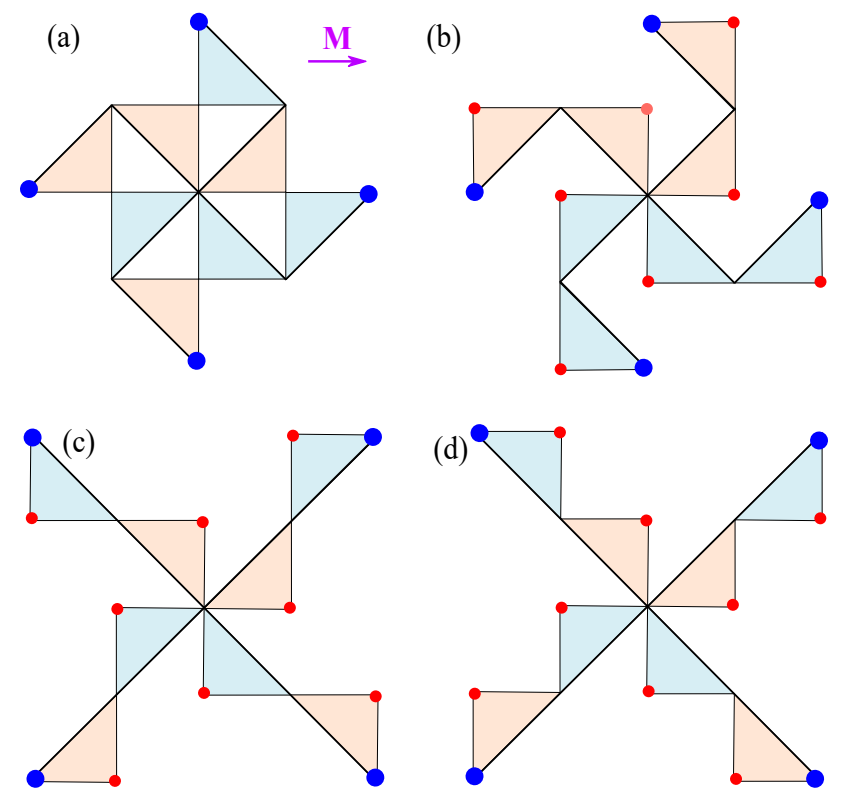

Fig. S14. Networks for MBS manipulation. The light blue and yellow color distinguish between two different phases of pairing potentials in the islands. The blue and red dots indicate the active and inactive MBSs, respectively. These setups are also scalable by adding more IRTIs to the "legs".

tential are likely, especially, around the boundary connecting to adjacent IRTIs. Therefore, in this section, we study the effect of such chemical potential inhomogeneity within each triangle. We focus on the junction between four triangles, considering all possible configurations of the chemical potentials. For simplicity, we use the parameter $w$ to model the width over which the chemical potential interpolates linearly between the values of adjacent triangles. Typical results are displayed in Fig. S15. In the left and center plots, the color indicates the chemical potential on each lattice site. The wavefunctions of MBSs are plotted on top. In the right plots, we show the low-energy part of the spectra as a function of $w$. As we can see, no important changes appear up to $w=10$ (the size of the triangles is 30 sites on the short sides). The wavefunction of MBSs seems to be barely affected, as well as the energy spectrum. Note that deviations from perfect degeneracy at zero energy can be exponentially suppressed by increasing the system size.

\section{Appendix E: Other alternative setups for braiding Majorana bound states}

In this section, we present some other simple network examples for Majorana manipulation, as shown in Fig. S14. Different from the one shown in the main text, we construct these networks by connecting the IRTIs only at the vertices. But similarly, two different phases of pairing potential (indicated by the cyan and yellow col- 

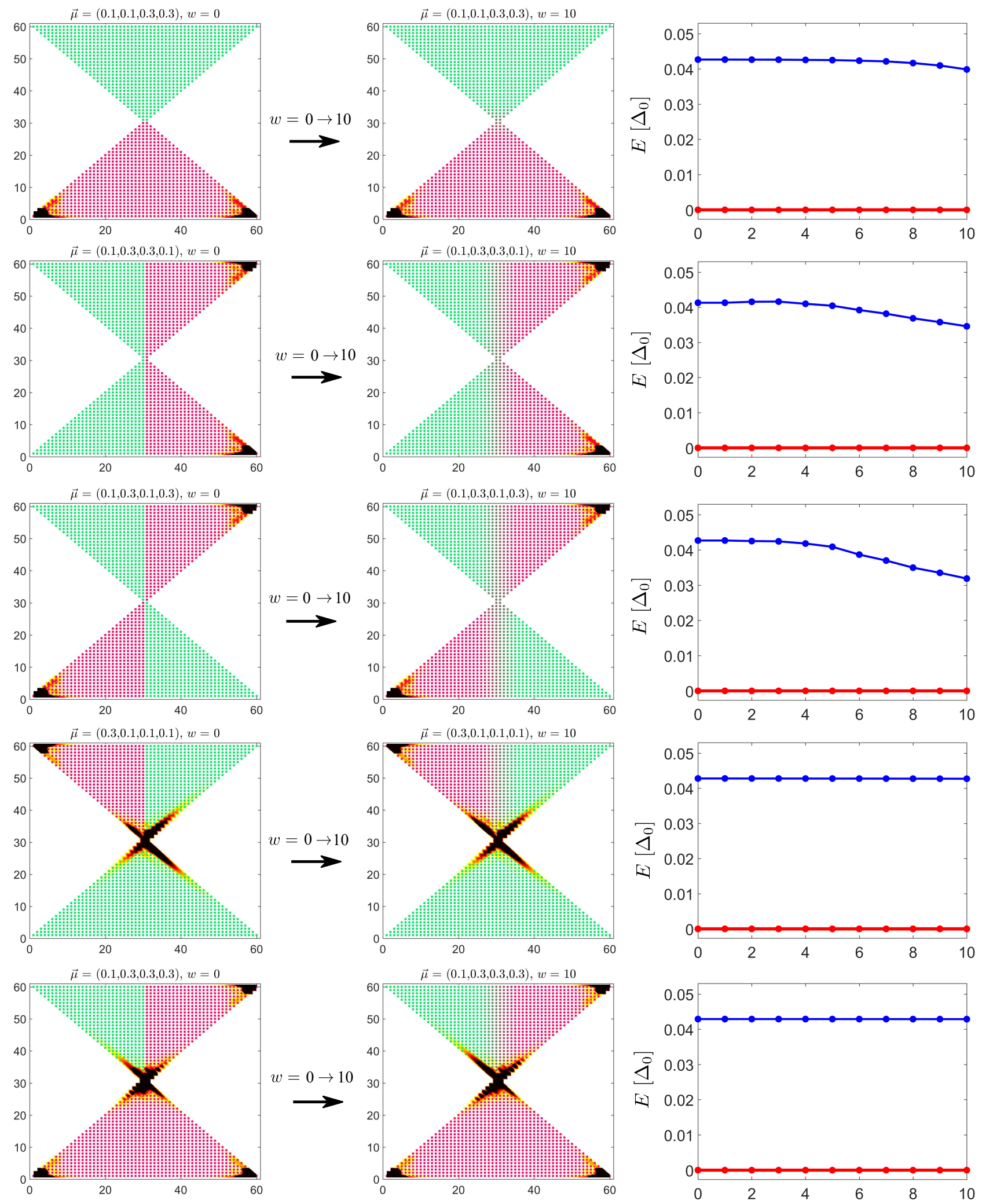

Fig. S15. Results in the presence of inhomogenous chemical potentials within individual IRTIs. The chemical potentials in the four triangles $\vec{\mu} \equiv\left(\mu_{1}, \mu_{2}, \mu_{3}, \mu_{4}\right)$ (in units of $\left.0.5 m_{0}\right)$ and the parameter $w$ are given in the titles of the panels in the first and second columns. The green-red color indicates the chemical potential on each lattice site (with the green limit for $\mu=\mu_{\mathrm{d}}$ while the magenta limit for $\mu=\mu_{\mathrm{u}}$ ). The panels in the third rows correspond to the energy spectra as functions of $w$. We can see clearly that increasing $w$ barely change the results. The MBSs stay robustly at the vertices and at zero energy. All the other parameters for all panels are the same as those in Fig. S6. 
ors) are applied in a strip form. The ferromagnetic of antiferromagnetic order ( $\mathbf{M})$ in the bulk is fixed and uniform. By locally controlling the chemical potentials in the IRTIs, we are not only able to increase or reduce the number of MBS pairs but also to control their positions. The Majorana qubits are also measurable via the Josephson effect or adjacent quantum dots, in a similar way we discussed before. Note that because the MBSs at the right-angle vertices of IRTIs are immobile, we can design our setups without connecting the right-angle vertices of IRTIs. In this case, extra MBSs appear at the unconnected right-angle vertices. However, they are "inactive" when performing braiding or measuring operations. 